\title{
AUTOMATIC FILTERING AND 2D MODELLING OF AIRBORNE LASER SCANNING BUILDING POINT CLOUD
}

Fayez Tarsha Kurdi*, Mohammad Awrangjeb and Nosheen Munir

Institute for Integrated and Intelligent Systems, Griffith University, Nathan QLD 4111, Australia

(f.tarshakurdi, m.awrangjeb)@griffith.edu.au; nosheen.munir@griffithuni.edu.au

\begin{abstract}
:
This paper suggests a new approach to automatic building footprint modelling using exclusively the airborne Light Detection and Ranging (Lidar) data. The first part of the suggested approach is the filtering of the building point cloud using the bias of the $\mathrm{Z}$ coordinate histogram. This operation aims to detect the points of roof class from the building point cloud. Hence, eight rules for the histogram interpretation are suggested. The second part of the suggested approach is the roof modelling algorithm. It starts by detecting the roof planes and calculating their adjacency matrix. Hence, the roof plane boundaries are classified into four categories: outer boundary, inner plane boundaries, roof detail boundaries and boundaries related to the missing planes. Finally, the junction relationships of roof plane boundaries are analyzed for detecting the roof vertices. With regard to the result accuracy quantification, the average values of the correctness and the completeness indices are employed in both approaches. In the filtering algorithm, their values are respectively equal to $97.5 \%$ and $98.6 \%$ whereas they are equal to $94.0 \%$ and $94.0 \%$ in the modelling approach. These results reflect the high efficacy of the suggested approach.
\end{abstract}

KEY WORDS: Filtering, Modelling, Segmentation, Lidar, Boundary, Point cloud 


\section{Introduction}

The requirement for automatic construction of 3D city models is increasing ceaselessly. That is why the acquisition of airborne laser scanning data has gained a strong position among the other data acquisition techniques. This system provides fast, day or night, 3D point cloud covering the scanned region. Once the Light Detection and Ranging (Lidar) technology started to become widespread, it was realized that the modelling of roof planes and their topological relationships is necessary for generating 3D building models. Therefore, significant efforts have been made towards this objective. There are two principal approaches for automatic generation of 3D building models. Firstly, the model-driven approach or parametric approach searches the most appropriate model from a set of basic building models contained in a model library. The second approach is the data-driven or non-parametric approach that calculates 3D models for buildings by using a series of more or less complex operations (see Tarsha Kurdi et al., 2007). Hence, the data-driven approach responds to extensive cases of building typology, whereas the model-driven approach stays limited for the given primitive buildings.

In urban areas, the point cloud of the city consists of several classes such as buildings, terrain, roads and vegetation. Regarding the difference between the conceptions of the employed modelling algorithm for each city class, it is necessary to extract each class separately before starting the modelling stage. The extraction of building class from the city scene can be achieved automatically or manually. Although the manual extraction is more accurate than the automatic one, the probability of obtaining a perfect result in the both cases is still limited. In this context, the majority of existing modelling (model driven or data driven) algorithms use the approximate hypothesis, which is not always realized, i.e., the building point cloud covers the roof surfaces (except for the vertical surfaces). In fact, the extracted building point cloud contains a considerable quantity of points which are not located on the roof. That is why the building points can be categorized into five classes which are the roof points (without the vertical surfaces), the roof vertical surface points, the facade points, the terrain points and the 
noisy points such as neighboring vegetation and birds. Except for the first class, all other classes are considered as undesirable points which may decrease the accuracy and generate deformation in the constructed building model. This issue requires the filtering of the building point cloud before starting the modelling procedure. The suggested algorithm in this paper depends on the analysis of a $Z$ coordinate histogram of a building point cloud. In fact, the point density of the roof facets is higher than the building facades. This hypothesis helps to develop eight rules for detecting the roof points from the building point cloud.

The question of roof plane boundary modelling presents a transition step between the detection of roof planes and the calculation of 3D building model. This procedure has not been given the position that it merits. Therefore, the goal of this paper is to focus on the automatic detection and modelling of $2 \mathrm{D}$ roof plane boundaries after filtering of the building point cloud. In this context, the result of the roof plane detection is expressed in raster form and then the roof topology is described by the plane adjacent matrix. Thereafter, the junction relationships between roof plane boundaries are analyzed to detect the roof vertices.

\section{Related work}

This section focuses on two topics: the automatic filtering of the building point cloud and the automatic modelling of inner roof plane boundaries.

\subsection{Building point cloud filtering}

The building point cloud may be classified into two main classes which are roof class and nonroof class. The roof class consists of a set of points which are used by the roof modelling algorithms, whereas the non-roof class represents a set of points which are undesirable and useless points for the roof modelling algorithms. Indeed, the majority of existing modelling algorithms use the approximate hypothesis that considers the building point cloud covering the roof surfaces (except for the vertical surfaces). Therefore, the non-roof class is named in this 
paper as the undesirable point class. Furthermore, the operation of roof class extraction from the building point cloud is referred to as filtering in this paper. Consequently, the undesirable point class (non-roof class) represents the terrain, facades, vegetation and noise.

Firstly, Tarsha Kurdi, Awrangjeb, and Munir (2019) calculate the building Digital Surface Model (DSM) by resembling that removes the undesirable points related to vertical elements such as building facades. Sometimes this operation does not eliminate all these points, which is why Park et al. (2019) eliminate the unnecessary objects by applying the cube operator to segment the building point cloud into roof surface patches, including superstructures, removing unnecessary objects, detecting the boundaries of buildings, and determining the model key points for building modelling.

For building detection by separating the off-terrain from the terrain, Demir (2018) studies the case of low terrain slope and detects the off-terrain objects via an elevation threshold. Thereafter, the non-building objects are eliminated because they have no planar features. Zhang, Yan, and Chen (2018) and Li, Rottensteiner, and Heipke (2019) use a region-growing algorithm based on the Triangulated Irregular Network (TIN) or grid data structures to detect the building roof point clouds.

In order to recognize the roof points, some authors use additional data such as the ground plan (Vosselman \& Dijkman, 2001) or the aerial images (Zhang et al., 2014).

For avoiding this issue, several approaches consider the input data is the roof point cloud instead of whole building point cloud (Jung \& Sohn, 2018; Awrangjeb, Gilani, \& Siddiqui, 2018).

Pirotti et al. (2019) test the use of TensorFlow neural network for deep learning to detect both the roof and the facade classes from Lidar point cloud. The extraction percentages of the roof and facade semantic classes respectively are 84\% and 76\%. Martin-Jimenez et al. (2020) remove the facade points by using the $Z$ component of the normal vector of each 3D point, 
where the covariance matrix of the eight neighboring points is analyzed and the normal vector of the adjusted surface is determined as the normal vector of the analyzed point.

\subsection{Modelling of inner roof plane boundaries}

For modelling inner roof plane boundaries, Rottensteiner et al. (2005) suggest taking into account the uncertainty of both the two neighboring planes and the approximate positions of the vertices of the polygon segments. Hence, statistical tests are introduced to minimize its dependence on the thresholds at all stages, including detection and classification of roof planes, boundaries, and step edges. Another approach by Ghaffarian et al. (2016) utilizes the Purposive Fast Independent Component Analysis algorithm (PFICA) for detecting roof plane boundaries, then employs the Douglas-Peucker algorithm after enhancing the obtained result by morphological filtering.

In the same context, the use of aerial images is suggested in addition to Lidar data. Schenk and Csatho (2002) detect roof plane boundaries from panchromatic images based on the texture discontinuity and utilize them to refine the results from Lidar data. Sohn and Dowman (2007) use the IKONOS multispectral images for roof plane boundary detection. Sampath and Shan (2006) detect roof planes and plane boundaries simultaneously by using exclusively Lidar data. Hence, they apply the linear space theory to separate plane boundaries from planar points. Gross and Thoennessen (2006) use the same principle for detecting the linear features from 3D point cloud. However, several authors suggest detecting firstly roof planes (Vosselman \& Dijkman, 2001). Then, they consider the set of roof planes as input for detecting plane boundaries. Sohn, Huang, and Tao (2008) apply region growing and TIN analysis for extracting roof planes. Then, Hough transform and neighboring plane intersection lines are applied in order to extract plane boundaries. Xiao et al. (2015) also start by extracting roof planes. Then, plane boundaries and vertices are calculated by using an undirected graph model of the building roof. Consequently, for obtaining reasonable and usable models with regular building boundary 
segments, the RANSAC (RANdom SAmple Consensus) algorithm is applied to find long segments and the corresponding parameters. Furthermore, other constraints are performed by applying parallel merging and orthogonal adjustments.

Goebbels and Pohle-Fröhlicha (2016) employ the raster form for detecting the main roof planes. Thereafter, the 2D-Hough-transform is applied for finding the step boundaries. The intersection boundaries are detected by using the Hesse normal forms and the Pavlidis' algorithm is applied in order to recognize the roof vertices. Starting from these vertices, the roof plane topology is described. At this stage, it is important to note that it is sometimes advised to start by modelling the outer building boundary. Awrangjeb (2016) suggests an algorithm for outer building boundary extraction. This algorithm has two major steps: outer boundary identification by using Delaunay triangulation and boundary tracking and smoothing before applying the least squares theory.

Some studies develop solutions for analyzing topological relationships between building roof planes. Ameri (2000) uses the Voronoï diagram of the roof planes. Jaya, Akshay, and Beena (2018), Xiong et al. (2015) and Perera, Nalani, and Maas (2012) use the roof topology graph based on the closed cycle graphs summarizing the topological relationships between the roof segments.

\section{Data}

In order to test our approach on different point densities, four types of data are used (see Table 1). 
Table 1. Characteristics of the four datasets used in this paper

\begin{tabular}{|c|c|c|c|c|}
\hline Hermanni & Strasbourg & Toronto & Vaihingen \\
\hline Acquisition & $\begin{array}{c}\text { End of } \\
\text { June 2002 }\end{array}$ & $\begin{array}{c}\text { Begin September } \\
2004\end{array}$ & February 2009 & 21 August 2008 \\
\hline TopoEye & $\begin{array}{c}\text { TopScan (Optech } \\
\text { ALTM 1225) }\end{array}$ & $\begin{array}{c}\text { Optech ALTM- } \\
\text { ORION M }\end{array}$ & $\begin{array}{c}\text { Leica } \\
\text { Geosystems } \\
\text { Leica ALS50 } \\
\text { system }\end{array}$ \\
\hline $\begin{array}{c}\text { Points } \\
\text { density } \\
\left(\text { points / } \mathrm{m}^{2}\right)\end{array}$ & $7-9$ & 1.3 & 6 & $4-6.7$ \\
\hline $\begin{array}{c}\text { Flight height } \\
(\mathrm{m})\end{array}$ & 200 & 1440 & 650 & 500 \\
\hline
\end{tabular}

The first test site, Hermanni, is a residential area in the periphery of Helsinki, where large and spaced out multi-storey buildings are surrounded by vegetation. This point cloud belongs to the building extraction project of EuroSDR (www.eurosdr.org). The second point cloud is of Strasbourg city in France. Several zones with different typology natures are covered, but point density and accuracy are lower in comparison to the Hermanni point cloud. The third point cloud is of Toronto city (Cramer, 2010). It contains representative scene characteristics of a modern mega city in Canada, including a mixture of low- and high-storey buildings with a wide variety of rooftop structures, streets and roads. The last dataset is captured over the city of Vaihingen in Germany (Cramer, 2010). This area is characterized by a few residential buildings, historic buildings and small-detached houses that are surrounded by trees.

The following section presents the filtering of building point cloud in the context of automatic detection of building roof point cloud.

\section{Filtering of building point cloud}

The airborne laser scanning in urban areas measures a 3D point cloud covering the scanned city. In order to model any building in the scanned city, the building point cloud first has to be extracted from the global point cloud. In order to achieve this purpose, two main ways are envisaged: automatic extraction and manual extraction. Despite the manual extraction being 
very slow compared with the automatic extraction, it is more accurate than the automatic extraction, especially if an expert in the Lidar data processing field is involved. Indeed, in the manual operation, the extraction is carried out per point basis and the operator may have extra information about the scanned scene such as aerial photos, city maps and/or other collected information, in addition to supplementary informatics navigation and visualization tools.

A building point cloud is a set of points that belongs to the same building. This definition is adopted in the general case for extracting the building point clouds from the point cloud of the city. At this stage, it is important to note that the elements presented by the building point cloud may be categorized into four classes. These classes are the roof points, the facade points, the building surrounding terrain points, and the noise points. In fact, the majority of modelling algorithms assume that all cloud points are distributed only on the building roof and all other points are considered as undesirable points. Moreover, the quantity of the undesirable points makes up a considerable proportion of the building point cloud and it perhaps generates deformation in the constructed building model. That is why it is necessary to detect the roof points before starting the modelling procedures.

In this paper, a new approach is suggested for achieving this goal. This algorithm is based on a $Z$ coordinate histogram analysis of a building point cloud. The basic hypothesis adopted in this approach is that the point density of the roof surfaces is very much higher than the building vertical surfaces such as the facades. That is why the $Z$ coordinate histogram interpretation allows detection of the roof point cloud. Indeed, the histogram represents the relationship between the building altitude gradation and the number of points, i.e., the histogram describes the point density in the function of the building altitude gradation.

\subsection{Analysis of $Z$ coordinate histogram of building point cloud}

The input of this algorithm is the extracted building point cloud which represents the roof, the facades, the surrounding terrain, small trees and vegetation attached to facades, and noise. The 
output is the roof point cloud. This algorithm starts by calculating the histogram of the $Z$ coordinate of the building point cloud (see Figure 1b). A histogram of a set with respect to a measurement represents the frequency of quantified values of that measurement among the samples (Serratosa \& Sanfeliu, 2006). A histogram of the $Z$ coordinate of building point cloud consists of a set of consecutive bars, each one representing the number of points within an altitude interval.

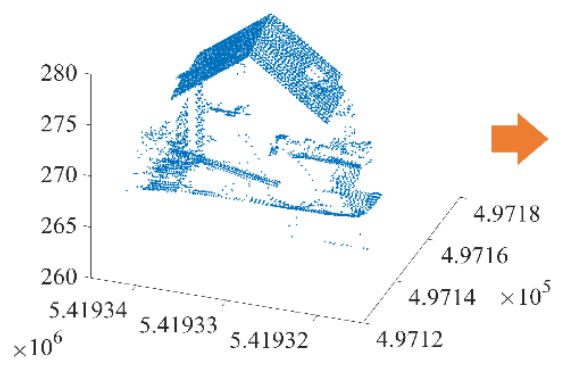

(a)

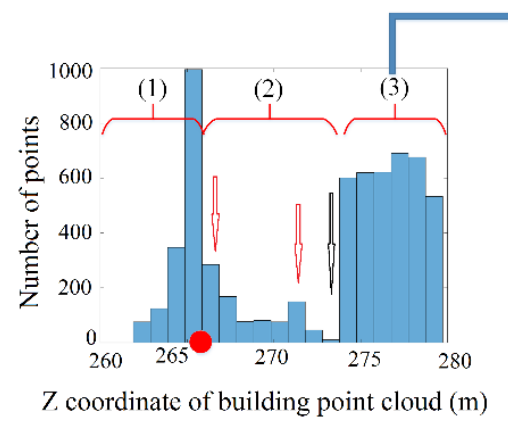

(b)

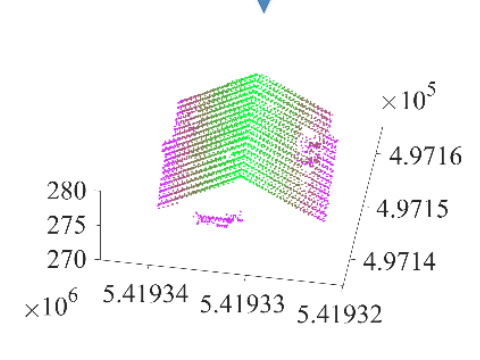

(c)

Figure 1. (a) Building point cloud; (b) Histogram of $Z$ coordinate of building point cloud; (c) Extracted building roof point cloud by using histogram. The building belongs to the Vaihingen site; color gradation in (c) represents the different bars in Section 3 of the histogram; Red circle in (b) represents the PSTF (Point Separating Terrain and Facades) sections.

From Figure 1, it can be noted that the histogram of the $Z$ coordinate of the building point cloud can be divided into three sections, where Section 1 represents the terrain surrounding building, small vegetation, noise and the lower parts of facades, Section 2 represents the points distributed on building facades, and Section 3 represents the roof point cloud. At this stage, an essential question arises: how can the limits among these three sections be determined? In order to answer this question, eight adopted rules will be presented later, but it is necessary at this point to explain the step and the orientation of the calculated histogram. 


\subsubsection{Histogram step}

The step value in the histogram is determined by considering the altitude interval that is occupied by the roof point cloud. Hence, several parameters are considered for calculating the histogram step. These include the altimetry accuracy of the point cloud, the roof texture thickness and the inclination of roof surfaces. Let us consider the case of a flat roof that consists of one plane. The altitude interval occupied by the roof point cloud is estimated as follows: $0.10 \mathrm{~m}$ (texture thickness) $+0.25 \mathrm{~m}$ (inclination of roof surface) $+2 \times 0.15 \mathrm{~m}$ (altimetry accuracy $)=0.65 \mathrm{~m}$. Unfortunately, the values of the first two parameters are not constant with all the scanned buildings in the same zone, so it is necessary to add a safety factor in order to ensure the presence of all roof points within one or maximum two bars. After a series of experiments, the step value is considered equal to $1 \mathrm{~m}$.

Contrariwise, though this step value sometimes allows localizing some facade points among the roof section, this tolerance will be accepted regarding the importance of safeguarding the roof points in the roof section. Finally, this implemented value of the histogram step may be used for most of the roof typology and point cloud density as illustrated in Figures 1 and 2.

\subsubsection{Histogram orientation}

The orientation of the calculated histogram is from left to right, that means the histogram represents the building point cloud from bottom to top. Consequently, the first bar in the histogram will represent the terrain surrounding the building and the last bar will belong to the building roof. This orientation explains the logical order of the three sections composing the histogram (see Section 4.1).

Once the step value and the orientation of the histogram are exposed, the rules of histogram division can be presented. 


\subsection{Rules of histogram interpretation}

As illustrated in Figure $1 \mathrm{~b}$, the histogram of the $Z$ coordinate of building point cloud can be divided into three main sections representing the terrain, the facades, and the roof. Moreover, the histograms of variant building point clouds are not identical (see Figure 2), with regard to the difference of building geometric forms and dimensions, the different characteristics of point clouds such as the point density, and finally the quantity and the distribution of undesirable points. Despite the difference between the building histograms, eight rules are adopted in order to interpret the majority of building histograms of the $Z$ coordinate, and consequently to divide them according to the composed sections:

Rule 1: The first bar of the histogram belongs to the terrain section.

Rule 2: The last bar of the histogram belongs to the building roof section.

Rule 3: This rule allows the determination of the PSTF (Point Separating Terrain and Facades). The red circle in Figure $1 \mathrm{~b}$ represents the PSTF. This point must be within the first four bars in the histogram. In fact, this value is considered after testing more than one hundred building point clouds of different point density, typology and topography. If there is a considerable number of terrain points in the building point cloud, as the cases of Figures 1a and 2a, the PSTF will be situated after the first bar, having considerable value (given threshold as described in Section 4.4) within the first four bars. Contrariwise, if there is not a considerable number of terrain points within the building point cloud as in the cases of Figures $2 \mathrm{~d}$ and $2 \mathrm{~g}$, the PSTF will be situated beside the fourth bar.

The importance of determining the PSTF arises from supposing that there are no roof planes in the bars preceding the PSTF. Even if it is sometimes possible to meet roof planes having low altitudes and small areas in this section, it will be hard to recognize them due to the high quantity of noisy points in this section. If there is a low quantity of points in the terrain section (Figures $2 \mathrm{~d}$, and $2 \mathrm{~g}$ ), then it will be possible to verify the presence of roof planes in the terrain section according to the suggested rules (see Section 4.3). 
Rule 4: Given that the point density of roof surfaces is significantly greater than that of facade surfaces, the fourth rule considers that each bar having considerable value (given the threshold as illustrated in Section 4.4) after the PSTF, belongs to the roof surface section.

Rule 5: The roof surface points may be presented in the histogram by one or several bars situated after the PSTF. All bars, after the last roof surface bar belong to the roof section (see Equation 4 in Section 4.4). These may represent the roof points, existing objects and/or details on the roof having considerable altitudes; such as chimneys, dormers, antenna and tanks, e.g., the green lift on the roof in Figure 2c is presented among the roof detail bars (see red arrow in Figure 2b).

Rule 6: The bar of the facade section that directly precedes the roof surface bars may represent some roof points in addition to the facade points (see the black arrow in Figure 1b). In order to avoid missing any roof point, the points belonging to the upper half part of this bar have to be added to the roof section. Though this operation may lead to adding some facade points to the roof section, it ensures never missing any roof point.

Rule 7: All undesirable point bars after the PSTF have small values regarding the values of roof surface bars, which is why the value of this threshold is assigned in function of the maximum value of roof bars (see Section 4.4).

Rule 8: In the histogram and after the PSTF, there may be bars having values smaller than the roof surface bars and greater than the undesirable point bars (see red arrows in Figure 1b). These bars represent fuzzy bars because these bars do not belong to the undesirable point or to the roof class.

In this context, it is important to point out that the fuzzy bars are situated after the PSTF which are why the second bar in Figure $1 \mathrm{~b}$ does not represent a fuzzy bar. There are two possibilities to explain the intermediary values of the fuzzy bars. They possibly represent vegetation, facade decoration or any other type of supplementary source of noise. The other 
possibility is the presence of small low roof planes in this zone because usually the planes situated down the mean level of the roof have small areas in comparison to the principal roof planes. In order to process this case and verify the presence of roof planes in the zone presented by the fuzzy bars, it is necessary to analyze the cloud points presented in this zone, and that will be detailed in the next section.

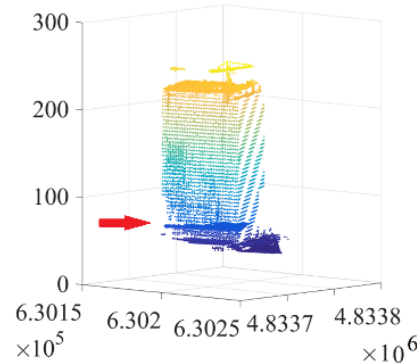

(a)

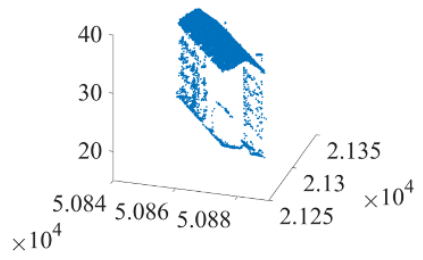

(d)

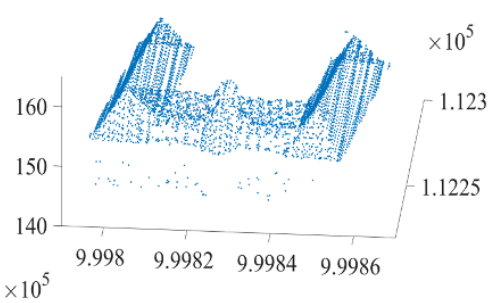

(g)

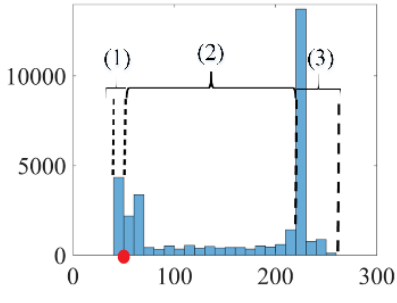

(b)

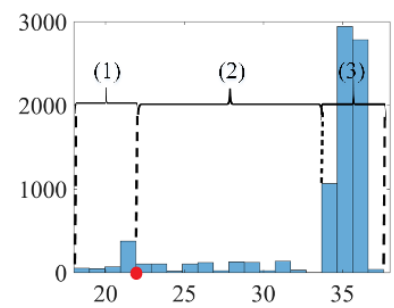

(e)

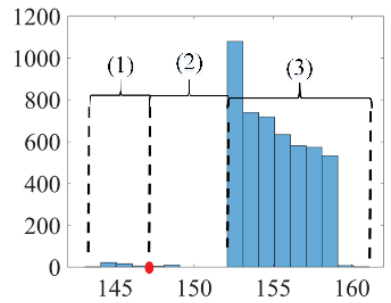

(h)

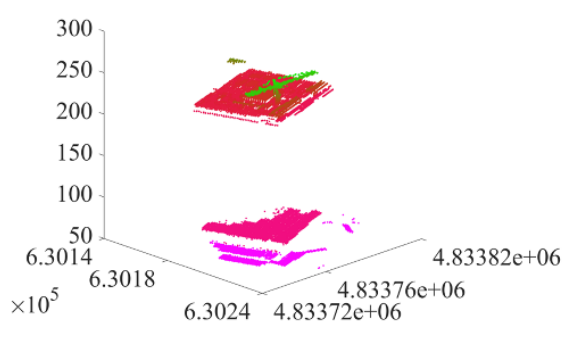

(c)

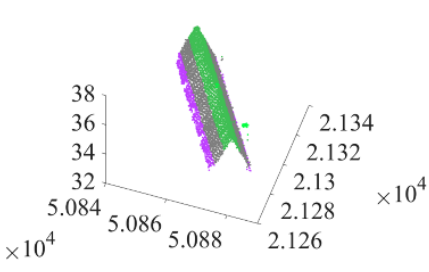

(f)

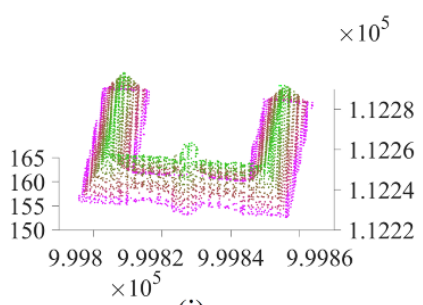

(i)

Figure 2. (a), (d) and (g) Point clouds of Buildings 1, 9 and 1 consecutivly in Toronto, Hermanni and Strasbourg datasets; (b), (e) and (h) Histograms of $Z$ coordinate of building point clouds consecutively of the last three buildings; (c), (f) and (i) Final results of building point clouds filtering; Red arrow in (b) points the bars of roof retails. 


\subsection{Analysis of histogram fuzzy bars}

As defined in the last section, fuzzy bars have values smaller than the roof surface bars and greater than the undesirable point bars (see red arrows in Figure 1b). Moreover, the presence of roof planes in the zone presented by these bars is possible. This section exposes how the fuzzy bars can be analyzed to save only the roof plane points.

Firstly, the cloud points corresponding the fuzzy bar have to be extracted from the building point cloud. Secondly, the extended RANSAC algorithm suggested by Tarsha Kurdi, Landes, and Grussenmeyer (2008) is adapted in order to reject the vertical planes because the researched roof planes are not vertical. Thereafter, this algorithm is applied for detecting the roof planes situated within the fuzzy bar zone. If it fails to detect any plane, that means the fuzzy bar does not represent any roof plane. However, in the case of successful plane detection, according to Tarsha Kurdi, Landes, and Grussenmeyer (2008), the application of the RANSAC algorithm sometimes allows the detection of planes which do not necessarily present roof planes. It represents perhaps one roof plane in addition to other noisy points which may belong to building facades (as the points inside the red circles in Figure 3d).

Furthermore, the detected plane may represent a group of noisy points fitting it. However, the detected plane has to be tested and the noisy points have to be eliminated from it by applying mathematical morphology procedures (the dilation and the erosion) on the binary DSM $(\mathrm{DSMb})$ calculated for the detected plane. Once this operation is achieved, if the detected plane represents a set of noisy points, distributed stochastically, then the plane is rejected. Otherwise, the points of the filtered detected plane are assigned to the final result.

Figure 3 presents the results of consecutive steps applied for filtering the building point cloud illustrated in Figure 3a. In this context, it underlines the results of the fuzzy bars analysis. At this stage, it is important to note that the fuzzy bars analysis is also applied in the building presented in Figure 1 and no planes are detected in the zones of the fuzzy bars. In the last two examples, the final results are almost identical to the reference roof point clouds. 


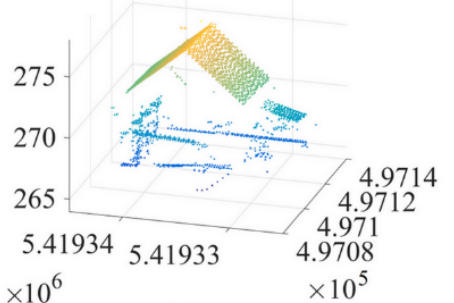

(a)

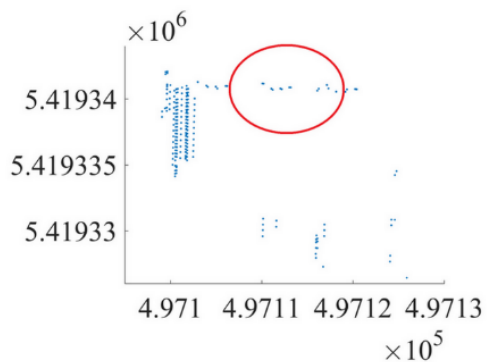

(d)

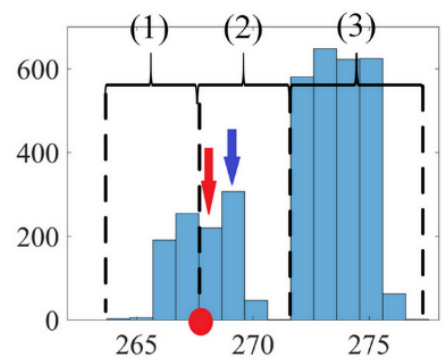

(b)

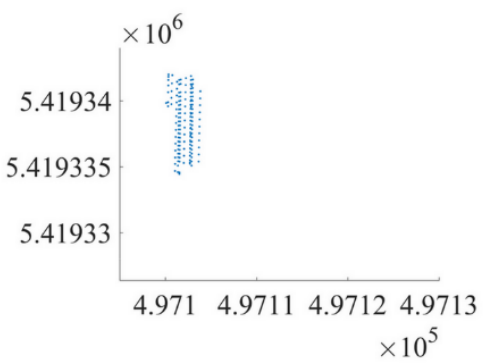

(e)

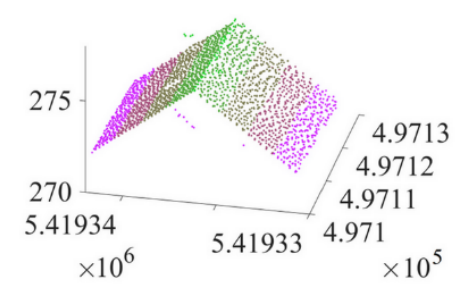

(c)

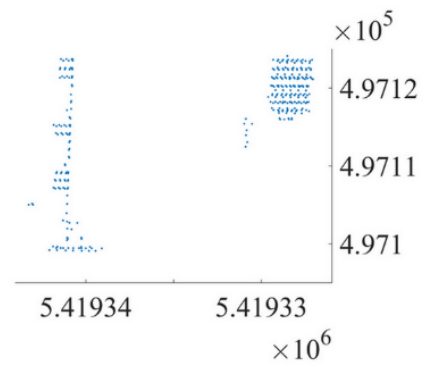

(f)

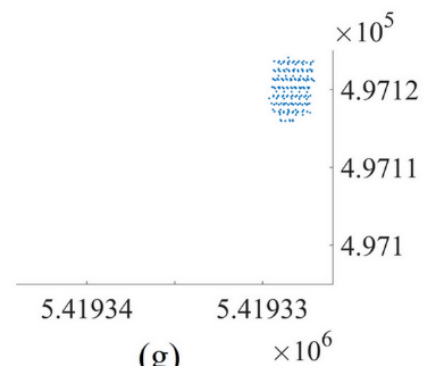

(g)

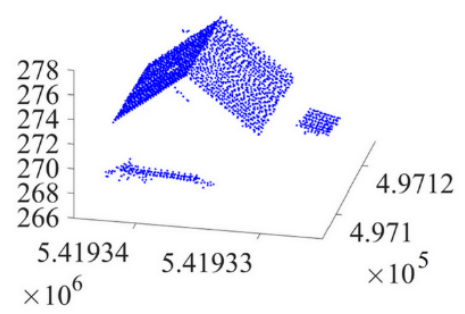

(h)

Figure 3. Analysis of fuzzy bars; (a) Point cloud of Building 17 in Vaihingen dataset; (b) Histogram of $Z$ coordinate of building point cloud; (c) Filtering result before considering the fuzzy bars; (d) and (f) Detected planes from the zone of fuzzy bars pointed to consecutively by red and blue arrows in (b); (e) and (g) Detected planes in (d) and (f) consecutively after deleting noise; (h) Final result of building point cloud filtering.

\subsection{Parameter setting}

There are three parameters used by the proposed approach; their values are chosen by the experiments through monitoring and evaluating the histograms of the $Z$ coordinate of almost 100 building point clouds with variant densities and urban typology (see Table 1). 
The first threshold is used in Rule 3 for determining the location of PSTF. As explained in Section 4.2, the PSTF is located within the first four bars in the histogram. If some of these bars have considerable values, the PSTF will be located next to the last one, otherwise it will be located by the fourth bar. The bar number $i$ is considered as the last bar of terrain section if Equation 1 is correct.

The second threshold is used in Rule 4 for detecting the bars representing the roof surface section where each bar after the PSTF having a considerable value (given the threshold) will belong to the roof surface section. In order to determine this threshold value, only the bars after the PSTF will be considered and a third of the maximum of the bar values is assigned to this threshold (see Equations 2 and 3).

$$
\begin{gathered}
h_{i}-h_{i+1}>0.5 \times h_{\text {max }} \text { or } h_{i}-h_{i+2}>0.6 \times h_{\text {max }} ; i \leq 4 \\
h_{m r b}=\max \left(h_{i}\right) ; i=\text { from PSTF to length }\left(h_{i}\right) \\
h_{r b}=h_{i} \geq \frac{1}{3} \times h_{m r b} ; i=\text { from PSTF to length }\left(h_{i}\right) \\
h_{l r b}=h_{r b}(\text { end })
\end{gathered}
$$

where $h_{i}$ is the value of the bar number $i, h_{m r b}$ is the greater roof bar value; $h_{r b}$ is one roof bar value; $h_{l r b}$ is the last roof surface bar and $h_{\max }$ is the maximum value of all histogram bars. The third threshold represents the maximum value of undesirable point bars in the histogram. This threshold is assigned in function of the greater roof bar value. Consequently, the value of the undesirable points bar has to be less than $10 \%$ of the greater roof bar value. In Figure 1b, the greater roof bar value is equal to 700 points, hence the threshold value is equal to 70 points. Once the filtering algorithm is presented, its efficiency will be tested, described in the next section.

\subsection{Accuracy of filtering algorithm}

In order to test the accuracy of the filtering algorithm, four datasets are used (see Section 3). The roof point clouds of the selected buildings in the four datasets are manually extracted (point 
per point), since manual extraction is supposed to be more accurate than automatic (Shan, Yan, \& Jiang, 2018) and then considered as references. In this context, it is beneficial to estimate the error of filtering. For this purpose, the confusion matrix is employed for calculating the correctness and the completeness accuracy indices (Shan, Yan, \& Jiang, 2018). In this context, three elements from the confusion matrix will be employed which are:

- TP (True Positive): the common points of both reference and detected roof

- FN (False Negative): the points of the reference not found in the detected roof

- FP (False Positive): the points of the detected roof not found in the reference

Table 2 represents the confusion matrix elements for estimating filtering accuracy of the Hermanni site buildings, i.e., TP, FN and FP values, in addition to the number of points of building point cloud before filtering, the undesirable point percentage, the Corr (Correctness) and the Comp (Completeness) values.

From Table 2, it can be noted that the undesirable point quantity in the building point cloud has considerable values (average $=13.37 \%$ ). The high efficiency of the suggested filtering algorithm can be observed from the values of Corr and Comp indices where they are almost equal to $100 \%$. Moreover, the visualization and comparison of the references and the obtained results show that the reason for the $\mathrm{FN}$ values being slightly greater than zero is not due to the filtering algorithm. The FN values are due to the accuracy of the manual extraction of the reference roof clouds. Indeed, in the manual extraction, the presence of confusing points attached to the transparent surfaces in the building, sometimes makes the operator not exactly sure of his decision.

Table 3 summarizes three tables similar to Table 2 for the three datasets of the Strasbourg, Vaihingen and Toronto cities. The correctness value for the Toronto data (94.33\%) is smaller than that of the other tested data (99.62\% and 98.45\%). In fact, as mentioned in Section 3, the Toronto data represents high-storey buildings with a wide variety of rooftop and facade 
structures and textures; e.g., in the building illustrated in Figures $2 \mathrm{a}, \mathrm{b}$ and c, a set of points of high density are detected inside the building (see the red arrow in Figure 2a). These points are perhaps due to the quality of facade texture on that level. On the one hand, as these points fit a plane and have a high density, they are detected by the filtering algorithm. On the other hand, for the operator who achieved the filtering manually, these points are confusing because it is unusual to find this quantity of points inside a building. To summarize, the cascading flat roof property and texture variety in the data for Toronto play a major role in this result.

However, despite the difference of the typology, the point density and the undesirable point percentage in these three point-clouds, the excellent obtained results refer to the capacity of the suggested filtering algorithm to process the majority of building and point cloud qualities.

Table 2. Confusion matrix elements for estimating filtering accuracy of Hermanni site buildings

\begin{tabular}{|c|c|c|c|c|c|c|c|c|c|}
\hline $\begin{array}{c}\text { Building } \\
\text { number }\end{array}$ & $\begin{array}{c}\text { Building } \\
\text { point } \\
\text { cloud }\end{array}$ & $\begin{array}{c}\text { Reference } \\
\text { roof } \\
\text { cloud }\end{array}$ & $\begin{array}{c}\text { Undesirable } \\
\text { points }\end{array}$ & $\begin{array}{c}\text { Filtered roof } \\
\text { cloud }\end{array}$ & TP & FN & FP & Corr & Comp \\
\hline \multicolumn{2}{|c|}{ (Points) } & $(\%)$ & \multicolumn{3}{|c|}{ (Points) } \\
\hline 1 & 6807 & 6388 & 6.16 & 6399 & 6375 & 13 & 24 & 99.62 & 99.8 \\
\hline 2 & 8272 & 7168 & 13.35 & 7153 & 7133 & 35 & 16 & 99.78 & 99.51 \\
\hline 3 & 3417 & 2931 & 14.22 & 2929 & 2921 & 10 & 8 & 99.73 & 99.66 \\
\hline 4 & 11153 & 8506 & 23.73 & 8498 & 8479 & 27 & 19 & 99.78 & 99.68 \\
\hline 5 & 7101 & 6338 & 10.74 & 6330 & 6324 & 14 & 6 & 99.91 & 99.78 \\
\hline 6 & 10455 & 8524 & 18.47 & 8601 & 8517 & 7 & 83 & 99.03 & 99.92 \\
\hline 7 & 8304 & 6836 & 17.68 & 6830 & 6818 & 18 & 12 & 99.82 & 99.74 \\
\hline 8 & 11132 & 9572 & 14.01 & 9581 & 9540 & 32 & 41 & 99.57 & 99.67 \\
\hline 9 & 4966 & 4382 & 11.76 & 4385 & 4375 & 7 & 10 & 99.77 & 99.84 \\
\hline 10 & 4419 & 4136 & 6.4 & 4142 & 4132 & 4 & 10 & 99.76 & 99.9 \\
\hline 11 & 5154 & 4609 & 10.57 & 4608 & 4604 & 5 & 4 & 99.91 & 99.89 \\
\hline \multicolumn{3}{|c|}{ Average } & 13.37 & & & & & 99.7 & 99.76 \\
\hline
\end{tabular}

TP: True Positive; FN: False Negative; FP: False Positive; Corr: Correctness; Comp: Completeness. 
Table3. Average values of three datasets filtering accuracy elements

\begin{tabular}{|c|c|c|c|c|c|}
\hline Dataset & $\begin{array}{c}\text { Number of } \\
\text { buildings }\end{array}$ & $\begin{array}{c}\text { Number of } \\
\text { points }\end{array}$ & $\begin{array}{c}\text { Undesirable } \\
\text { points } \\
(\%)\end{array}$ & Corr $(\%)$ & $\begin{array}{c}\text { Comp } \\
(\%)\end{array}$ \\
\hline & & \multicolumn{5}{|c|}{ Average values } \\
\hline Strasbourg & 14 & 3438 & 7.31 & 99.62 & 99.65 \\
\hline Toronto & 7 & 36682 & 37.41 & 94.33 & 97.23 \\
\hline Vaihingen & 30 & 3100 & 36.59 & 98.45 & 98.92 \\
\hline
\end{tabular}

Corr: Correctness; Comp: Completeness.

With the filtering algorithm exposed and tested, the following section presents a new approach to automatic calculation of 2D building models starting from Lidar building point cloud.

\section{Automatic calculation of $2 \mathrm{D}$ building models}

As discussed in Section 2.2, Goebbels and Pohle-Fröhlicha (2016) employ the raster form for constructing the $3 \mathrm{D}$ building model from Lidar data. Though the suggested approach in this paper depends also on the raster form for calculating the building footprint, it differs from it in several points as presented in Table 4. 
Table 4. Main differences between the suggested approach and that suggested by Goebbels and Pohle-Fröhlicha (2016)

\begin{tabular}{|l|l|l|}
\hline & Suggested approach & $\begin{array}{l}\text { Goebbels and Pohle- } \\
\text { Fröhlicha (2016) approach }\end{array}$ \\
\hline Plane detection & Extended RANSAC & $\begin{array}{l}\text { Segmenting angles of } \\
\text { gradients and RANSAC }\end{array}$ \\
\hline Inner boundary detection & $\begin{array}{l}\text { Directly from building label } \\
\text { image }\end{array}$ & $\begin{array}{l}\text { 2D-Hough-transform and } \\
\text { Hesse normal forms }\end{array}$ \\
\hline Outer Boundary detection & $\begin{array}{l}\text { Directly from building label } \\
\text { image }\end{array}$ & Taken from cadastral data \\
\hline Roof topology & -Before vertices detection & $\begin{array}{l}\text {-After vertices detection } \\
\text {-Analysis of vertex } \\
\text { neighborhood }\end{array}$ \\
\hline Roof details & Alane adjacent matrix & Are not considered \\
\hline Missing details & Are considered & Are not considered \\
\hline Non-planer surfaces & Are not considered & $\begin{array}{l}\text { With the exception of } \\
\text { rotation bodies, non-planar } \\
\text { surfaces are not dealt with }\end{array}$ \\
\hline
\end{tabular}

The first step in the automatic construction of 2D building footprint models, starting from Lidar building roof point cloud, is the calculation of the building label image. This image will be the input data for detecting and modelling roof plane boundaries.

\subsection{Calculation of building label image}

In order to automatically detect the roof planes, the RANSAC algorithm was extended to exceed its limitations. Its major limitation is that it searches to detect the best mathematical plane among 3D building point cloud even if this plane does not always represent a roof plane. The proposed extension allows harmonizing the mathematical aspect of the algorithm with the geometry of a roof. At this stage, it is important to note that the building point cloud is the input of the extended RANSAC algorithm. The final result of roof plane detection is a building label image which represents the building roof in 2D. This image is segmented according to the roof planes (see Figure 4a). For more details about the extended RANSAC algorithm see Tarsha Kurdi, Landes, and Grussenmeyer (2008). Once the building label image is calculated, the 
missing roof planes have to be detected and then the automatic modelling of roof plane boundaries can be started.

\subsection{Detecting and modelling missing roof planes}

Unfortunately, the calculated building label image in the last section does not represent all building roof details. Some roof planes with small areas are missed. The missing plane points are considered in the last section as undesirable noise. Consequently, the detection of these planes will not only allow the presenting of all roof planes, but it will also improve the accuracy of the calculated building label image because the missing plane points will not play the role of undesirable noise. In fact, the missing planes are due to the numerous items that may be located on a building roof such as dormers, chimneys, antennas, decorative features, and other objects. Thus, they have irregular geometric forms. Moreover, there are two types of missing roof planes on building roofs: firstly, the details having considerable altitudes and negligible areas, and secondly the details having negligible altitudes regardless of the area values. In this paper, the approach suggested by Tarsha Kurdi and Awrangjeb (2020) for modelling the missing planes is employed. This approach allows only modelling the missing roof planes having considerable altitudes since it depends on the height of the missing roof planes in the error map matrix (see Figure 9a). Therefore, only error map matrix pixels having values greater than $+0.5 \mathrm{~m}$ are considered. The analysis of the error map matrix by considering this rule allows the calculation of a new matrix named the mask of missing roof planes.

In order to detect the missing roof planes, a region-growing algorithm is applied on the missing plane mask. This procedure allows the independent detection of each missing roof plane. Because of the negligible areas of these planes, their geometric forms appear vague in the mask. Therefore, each plane will be presented by a square situated in the gravity center of each roof plane. Moreover, its area is equal to two pixels. For more details about this algorithm see Tarsha Kurdi and Awrangjeb (2020). 


\subsection{Modelling of outer roof boundary}

The roof plane boundaries consist of two categories which are the outer roof boundary and the inner roof plane boundaries. From Figure 4a, it can be observed that one pixel of the outer roof boundary is a non-zero pixel and at least one of its eight neighboring pixels is equal to zero. This observation enables the easy detection of the pixels belonging to the outer roof boundary. Once the outer roof boundary polygon is detected and filtered, the Douglas-Peucker technique is employed for calculating the extremity point coordinates as well as the list of all points describing every outer boundary segment line. After that, the least squares theory is applied to calculate the mean line of each segment. Finally, the outer roof boundary is enhanced by merging the boundary segments that belong to the same facade plane. For more details about this step, see Tarsha Kurdi, Landes, \& Grussenmeyer (2007). The result of this operation defines a new matrix named $O_{\mathrm{b}}$ (Outer boundaries) matrix (see Figure 4d).

\subsection{Modelling of inner roof plane boundaries}

After calculating the building label image and modelling the outer roof boundary polygon, the next step toward 2D roof modelling is the detection of the inner roof plane boundaries. In the literature (Ameri, 2000; Rottensteiner, 2003), it is suggested to use Voronoï diagram to achieve this task. However, this solution is unsatisfactory because it creates distortions not only on the actual position of the plane boundaries, but also on adjacency relationships between planes. Furthermore, Vosselman and Dijkman (2001) suggest calculating the intersection between the adjacent planes for detecting the plane boundaries. In fact, the outer boundaries and the step edges cannot be obtained from the plane equations and they are much more difficult to find. Consequently, the Vosselman and Dijkman (2001) solution is not advised. This sometimes generates undesirable deformations in the level of 2D building models. Therefore, a new method is proposed for constructing a more reliable model. This method detects the inner roof 
plane boundaries directly from the building label image. This choice is adopted because the building label image is calculated and corrected precisely (see Sections 5.1 and 5.2).

The input data for detecting the inner roof boundaries is the building label image, which presents the segmented roof. In order to detect the inner roof plane boundaries, there are four successive steps: identification of the adjacency relationship between neighboring planes, detection of roof plane boundaries, analysis of the junction relationships between boundaries and detection of roof vertices.

\subsubsection{Adjacency relationship between roof planes}

In order to identify the neighborhood relationships between roof planes, an upper triangular binary matrix $M=\left[m_{i, j}\right]$, which is called the neighborhood matrix or the so-called plane adjacent matrix, is calculated. In $M, m_{i, j}=\{0,1\}, i \leq j$ and $1 \leq i, j \leq n$, where $i$ and $j$ indicate row and column indices, respectively, and $n$ indicates the number of detected roof planes, e.g., in the building presented in Figure $4 \mathrm{a}$, since there are 7 roof planes $n=7$ and $M$ is a $7 \times 7$ square matrix. Since, $M$ is an upper triangular binary matrix, when Planes 1 and 2 are adjacent, we put $m_{1,2}=1$ and $m_{2,1}=0$

In order to fill $M$, the following operation is repeated for each roof plane, i.e., Plane 2 in Figure $4 \mathrm{a}$ is considered. The building label image matrix presented in Figure $4 \mathrm{a}$ is named $S_{\mathrm{i}}$ (Segmented image). At this stage, the binary image of Plane 2 named $P_{2}$ matrix, is calculated. Thereafter, the negative image of $P_{2}$ named $N_{\mathrm{p} 2}$ is calculated. Then, a band of pixels around Plane 2 is added to the matrix $P_{2}$ defining a new matrix named $E_{\mathrm{p} 2}$ (Extended Plane 2). Finally, a new matrix named $A_{\mathrm{p} 2}$ (Adjacency of Plane 2) is defined (see Equation 5). This new matrix allows the determination of all adjacent planes of Plane 2. Then, it allows the filling of the part concerning this plane in the $M$ matrix.

$$
A_{\mathrm{p} 2}=E_{\mathrm{p} 2} \times N_{\mathrm{p} 2} \times S_{\mathrm{i}}
$$


Where $\times$ indicates element by element multiplication.

In order to fill $M$, the last operation is repeated for each roof plane. The pseudocode below explains this operation:

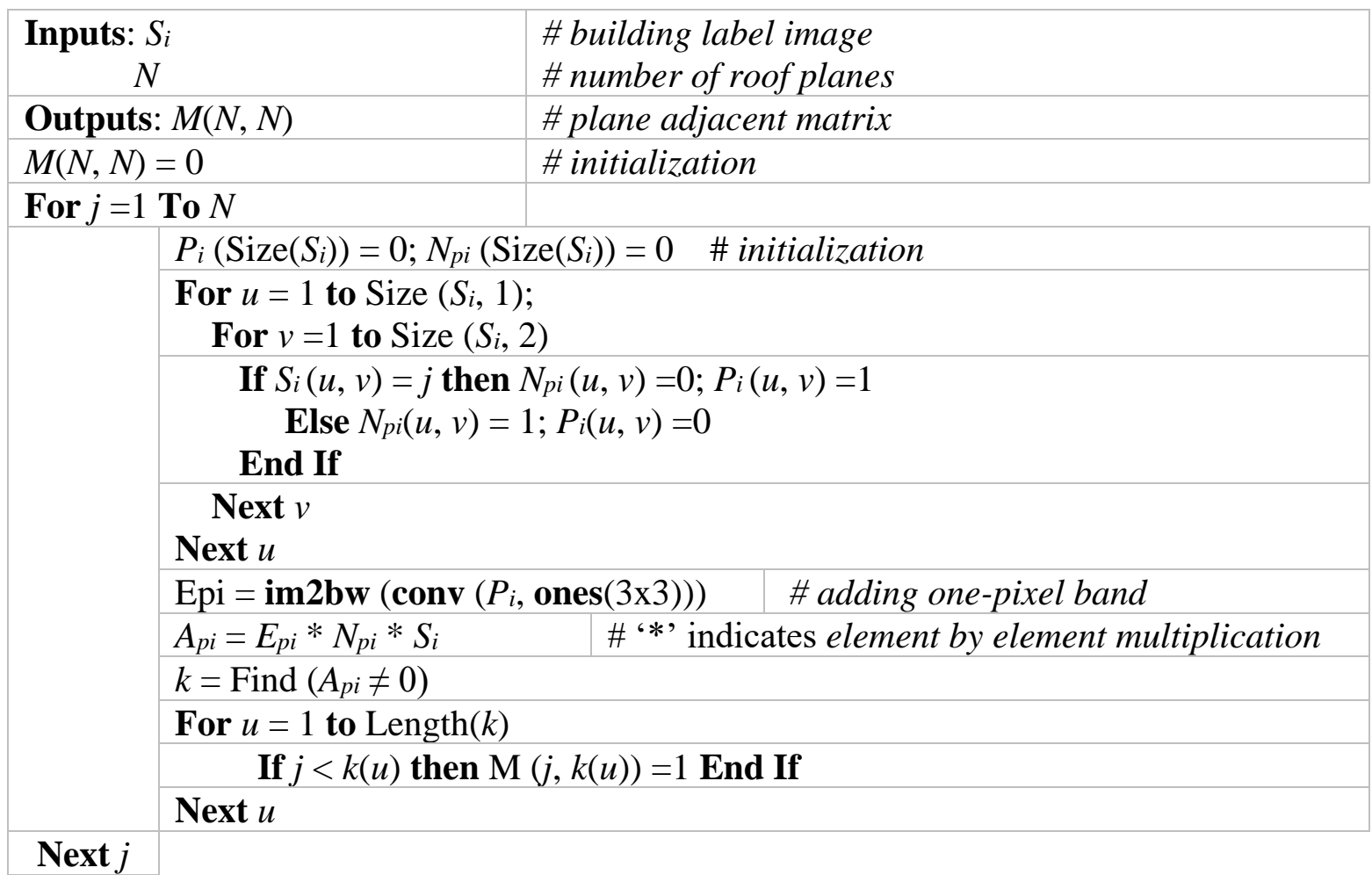

In this pseudocode, the comments are in italics, conv is a $2 \mathrm{D}$ convolution with all ones $3 \times 3$ moving window; im2bw: is a function that transforms a matrix into a binary matrix.

Once $M$ is completely calculated, the step of inner roof plane boundary detection is started. 


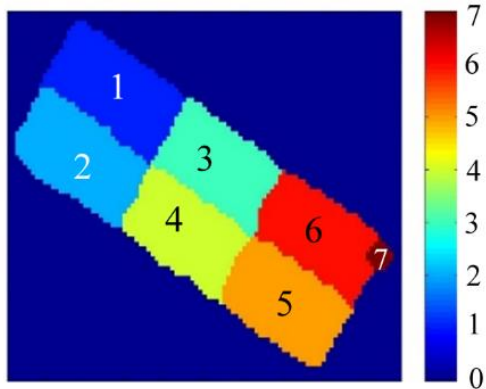

(a)

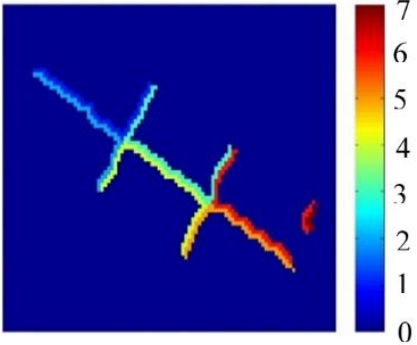

(b)

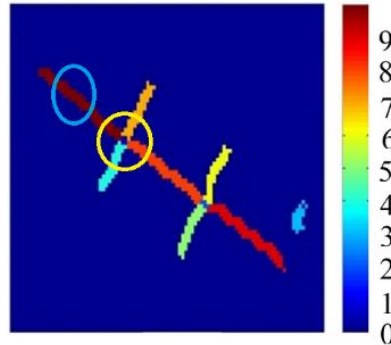

(c)

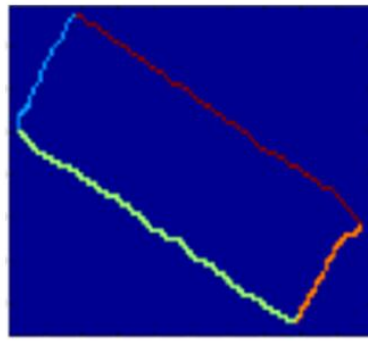

(d)

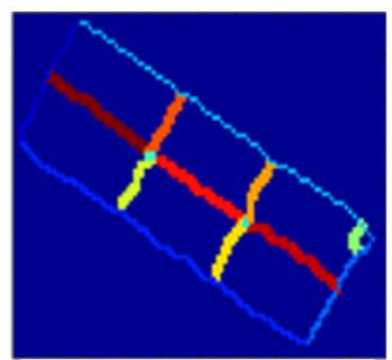

(e)

Figure 4. Modelling of inner roof plane boundaries. (a) Building label image; (b) Localization of pixels of inner plane boundaries (c) Visualization of $R_{\mathrm{ib}}$ (Raster inner boundary) matrix; (d) Visualisation of $O_{\mathrm{b}}$ (Outer boundaries) matrix; (e) Visualisation of $A_{r b}$ (All roof boundaries) matrix; Colors in (a) and (b) represent the plane numbers, colors in (c), (d) and (e) represent the boundary numbers.

\subsubsection{Detection of inner roof plane boundaries}

In the building label image, the inner plane boundaries are represented by the pixels located on the border between the adjacent planes. The first step is to detect these pixels. For this purpose, a $3 \times 3$ moving window is used for testing the vicinity of each pixel. This operation allows localizing pixels of inner plane boundaries (see Figure 4b). At this stage, in the image processing field, several software libraries such as OpenCV (Open Computer Vision) are available. These libraries offer standard functions that can be used for achieving this procedure. In Figure 4b, it can be noted that the boundary of two adjacent planes is defined by two-pixel rows, one belongs to the first plane and the other belongs to its neighbor. Therefore, the value 
of each pixel is the number of the original plane. Then, these pairs of pixel rows are merged to give two pixel-rows of the same value which is the boundary segment number. Figure $4 \mathrm{c}$ represents the visualization of matrix $R_{\mathrm{ib}}$ (Raster inner boundary), in which the pixel values represent the boundary segment numbers.

In $R_{\mathrm{ib}}$ matrix, two kinds of pixels are met: pixels of boundary between two adjacent planes (boundary circled in blue in Figure 4c), and pixels located at the intersection of more than two planes (boundary circled in yellow in Figure 4c). The latter pixel seems the same as a vertex rather than a boundary segment. But it is considered as a boundary segment because it appears in the $M$ matrix. That is why it is named a punctual boundary. To localize these pixels, it suffices to note that they appear twice in the $M$ matrix and each pixel has a minimum of three neighboring planes. In Figure 4c, there are four neighboring planes of the boundary circled in yellow.

Once the matrix $R_{\mathrm{ib}}$ is calculated, the plane boundaries are localised and saved as a new list. It can be observed that one boundary segment is defined by two adjacent pixel rows; each one belongs to a different plane. To limit them as one row, the median row is calculated. Finally, another problem met with frequently during the $2 \mathrm{D}$ roof modelling, is the presence of roof details located on the inner plane boundaries, e.g., fireplace on the boundary. In this case, despite the boundary between the two planes being defined only one time in $M$ matrix, it is decomposed into two portions. For this purpose, a region-growing algorithm is applied to detect each portion independently.

After the detection of the inner roof plane boundaries, the automatic detection of the roof vertices is initiated. For carrying out this procedure, the junction relationships between the boundaries have to be studied. 


\subsubsection{Analysis of junction relationships between boundaries}

Since the processing is in $2 \mathrm{D}$, a vertex means the junction point of two boundaries at least. The importance of analyzing the junction relationships between boundaries comes from the fact that a vertex is located at the junction of several boundary extremities. But the vertex coordinates $X$ and $Y$ will not be exactly the same for all boundary extremities. That is why it must be possible to find out how to join the boundary extremities. The innovative idea in this contribution is to describe the junction relationships between boundaries through a boundary number in a specific order. For example, if all boundaries surrounding one plane are taken together, then each boundary corresponds to an order of succession. The proposed algorithm begins by highlighting all roof boundaries, both inner boundaries and outer ones. This leads to the creation of a new matrix $A_{r b}$ (All roof boundaries) obtained by adding the $O_{\mathrm{b}}$ matrix to the $R_{\text {ib }}$ matrix (see Figure 4e).

Before presenting an example, it is important to explain the method of computing the junction relationship between the boundaries enveloping one plane. Starting from the $A_{r b}$ matrix and by using the binary matrix of selected plane named $B_{\mathrm{p}}$ (Binary plane) matrix, the boundaries enveloping this plane matrix that are named $E_{\mathrm{b}}$ (Enveloping boundaries) matrix, can be detected by multiplying the last two matrixes element by element, as shown in Equation 6 .

$$
E_{\mathrm{b}}=A_{\mathrm{rb}} \times B_{\mathrm{p}}
$$

where $« X »$ is the multiplication element by element

In the matrix $A_{r b}$, the numeration of the outer building boundaries starts from 1 to $m$; where $m$ is the number of outer building boundaries. After that, the numeration of the inner building boundaries starts from $m+1$ to $m+s$; where $s$ is the number of inner roof boundaries (see Figure 4e).

At this stage, the analysis of the junction relationships between boundaries can be started. For this purpose, the junction relationship between boundaries enveloping each plane is calculated. 
Consequently, $n$ junction relations are obtained, where $n$ is the number of roof planes. Finally, global analysis of all junction relationships allows the determination of the boundary which passes through each vertex. The roof vertices are also detected and assigned.

From the $E_{\mathrm{b}}$ matrix which presents the boundaries enveloping Plane 2 in Figure $5 \mathrm{~b}$, the junction relationship between boundaries is deduced as the following: 1, 2, 7, 12 and 5. This junction relationship presents the order of the boundaries enveloping Plane 2. In the same way, a new junction relationship can be deduced for each roof plane. Moreover, each junction relationship generates one or several triples, e.g., the junction relationship of Plane 2 in Figure $5 b$ generates the following triples: $(1,2,7),(2,7,12),(7,12,5),(12,5,1)$ and $(5,1,2)$. Let us take the triple $(7,12,5)$. It means that Boundaries 7 and 12 pass through the same vertex. Furthermore, Boundaries 12 and 5 pass also through the same vertex. To conclude, the analysis of all roof plane triples allows detection of all boundary extremities forming each vertex. Furthermore, it allows for distinguishing the different vertex classes as it will be presented in Section 5.4.4. Once the inner roof boundaries are detected and all roof planes assigned, the detection of roof vertices can be carried out as shown in the next section.

In order to detect the vertices, it is necessary to distinguish between planes having a single adjacent plane, named "plane of single adjacency", e.g., Planes 1, 2 and 3 in Figure 5a, and planes having more than one adjacent plane named "plane of multiple adjacencies", e.g., Planes 1, 2, 3, 4, 5 and 6 in Figure $5 b$.

The two plane types can be distinguished directly from the plane junction relationship. Indeed, if a plane shares exclusively its boundaries with another plane as Plane 1 in Figure 5a, then, the plane is a "single adjacency", e.g., Planes 2 and 3 in Figure 5a. In all other cases, the plane is a "multiple adjacencies", e.g., Planes 1, 2, 3, 4, 5, and 6 in Figure 5b. At present, it must similarly distinguish between two types of inner roof plane boundaries. The first one is a boundary that envelops a single adjacency plane and the second one is a boundary that envelops 
a "multiple adjacencies" plane. This boundary type is named boundary of type $S$ and the other boundary type is named boundary of type $M$. Likewise, when a vertex joins boundaries of type $S$, then it is named vertex of type $S$, and when a vertex joins boundaries of type $M$, then it is named vertex of type $M$.

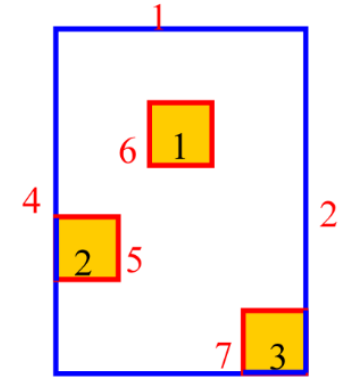

3

(a)

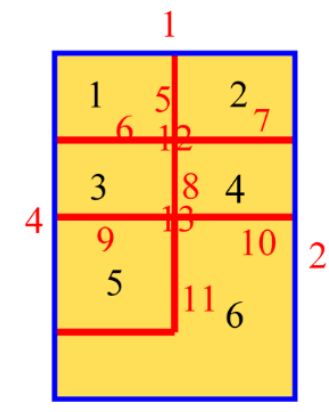

3

(b)

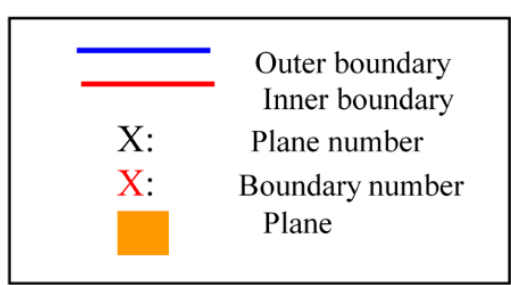

Figure 5. Representation of two roof planes types; (a) Representation of single adjacency planes; (b) Representation of multiple adjacency planes.

Once the boundaries are detected and distinguished by their types, the vertices detection can be achieved.

\subsubsection{Detection of roof vertices}

Since, a vertex combines two or more boundaries, the boundary extremities passing through the same vertex must have the same coordinates $X$ and $Y$. However, it is not the case for the detected boundaries discussed in Section 5.4.3. Hence, in order to merge all potential vertices into a single one, it is first necessary to detect them. Previously, two vertex types were defined depending on the type of adjacent planes. These vertex types are the vertex of type $S$ and the vertex of type $M$. In the following, methods of automatic detection of each vertex type are exposed. 
Automatic detection of vertices of type $M$ : It is shown above the junction relationship of each plane can be presented by a set of triples. Each one is a suite of three boundaries. Consequently, it defines two vertices. For example, the triple $(7,12,5)$ presents two vertices: $(7,12)$ and $(12$, 5) (see Figure 5b). After detection all roof triples, then it can be noted that one vertex can be presented within several triples.

Automatic detection of vertices of type $S$ : It has been seen that in the boundary of type $S$, the junction relationship contains only one inner boundary. In this case, the plane area (in pixel unit) becomes a criterion for decision. Empirically, the employed value of this threshold is equal to 12 pixels. Indeed, if the roof detail area is smaller than a given threshold, then its geometry may become vague. Moreover, the threshold is taken in pixel unit because it relates to the point density. If the plane area is greater than the given threshold, it is possible to apply the Douglas-Peucker algorithm to decompose the plane outline polygon according to its sides. In this case, the employed threshold in the Douglas-Peucker algorithm is equal to 2.5 pixels. Otherwise, if the plane area is smaller than the given threshold, it is necessary to distinguish two kinds of planes of type $S$. The first one is the case of a junction relationship consisting of one inner boundary only (Plane 1 in Figure 5a). The second kind is the case of a junction relationship containing several boundaries but only one among them is an inner boundary (Planes 2 and 3 in Figure 5a). This distinction is made because each one of the two plane kinds has a specific manner of processing.

- Case of junction relationship consists of one boundary only: Let us take the example of Plane 1 in Figure 5a. On the one hand, the junction relationship is composed of a single boundary according to the boundary definition (a set of pixels describing the same adjacency relationship between two planes), but it has, on the other hand, four vertices (the plane outline polygon corners). In this case, geometric constraints are introduced by assuming the geometric plane form is rectangular (because its geometry in building label image is vague), which is the 
most frequent case. Hence, the coordinates of the four rectangle corners (vertices) are calculated by using the modelling method based on static moments. All details and equations of this method are given by Tarsha Kurdi et al. (2007) in the context of the parametric modelling approach of building footprint.

- Case of junction relationship containing several boundaries: Taking the example of the Planes 2 and 3 in Figure 5a, two different situations arise: either the plane is attached to a single outer boundary as Plane 2, or the plane is adjacent to two outer boundaries as Plane 3. In the first case, the plane is defined by three inner boundaries and four vertices. In the other case, while the plane is adjacent to two outer boundaries, it is defined by two inner boundaries and three vertices. Vertex calculation follows a process that studies the angle formed by two successive outer boundaries of the considered plane. This hypothesis can be explained by the fact that it is possible to meet a plane adjacent to two outer boundaries but the angle between the two outer boundaries is close to $180^{\circ}$ (see Figure 6c). Despite the plane being attached to two outer boundaries, its model is similar to the case of the plane attached to a single outer boundary. In order to distinguish between the two cases, the farthest pixel, among the plane perimeter ones, from the plane gravity center has to be detected. This point and the plane gravity center define a circle radius. The center of this circle is the plane gravity center. Then, we calculate the intersection of this circle with the outer boundaries that surround the plane (see points $\mathrm{C}$ and $\mathrm{D}$ in Figure 6c). 


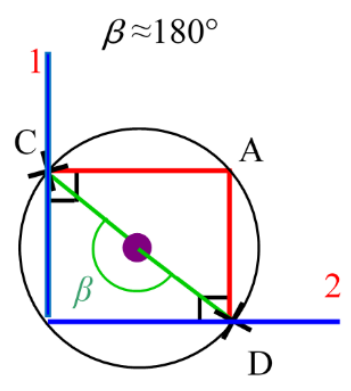

(a)

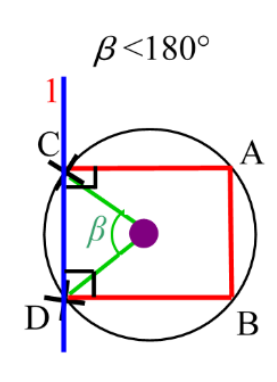

(b)

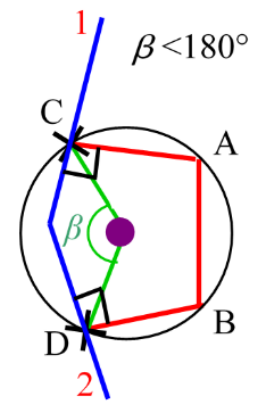

(c)

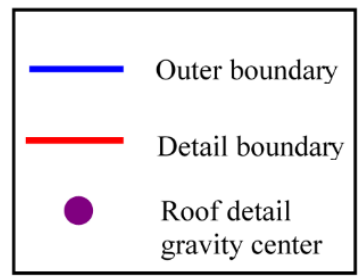

Figure 6. Modelling of roof planes of type $S$ attached to building outer boundary; (a) Plane attached to two outer boundaries; (b) Plane attached to a single outer boundary; (c) Plane attached to two outer boundaries but it is treated as the case of (b).

In Figure 6, the angle $\beta$ is calculated from the center of gravity and the two last points $\mathrm{C}$ and D. If this angle $\beta$ is close to $180^{\circ}$ as in the case of Figure $6 a$, then the plane is considered as attached to two outer boundaries, otherwise the plane is taken as attached to one outer boundary (see Figures $6 \mathrm{~b}$ and $6 \mathrm{c}$ ). Finally, two perpendicular lines are drawn from the intersection points C and D. If our case corresponds to Figure 6a, then the intersection of the two perpendicular lines is calculated. Otherwise, the intersection points of the two perpendicular lines with the circle are calculated.

At this stage, all inner roof plane boundaries are extracted. In this context, one inner roof boundary is a list of connected pixels linking two consecutive vertices. One inner boundary may sometimes be composed of several straight-line segments (Boundary 11 in Figure 5b). Hence, all the extracted boundaries have to be tested by applying the Douglas-Peucker technique. This procedure allows the inner boundary segments to be decomposed according to the lines. At this phase, the fundamental elements, which are necessary for calculating the $2 \mathrm{D}$ building roof model, are available. 


\subsection{Results}

As mentioned above, the input data in the suggested algorithm is a building point cloud which is free of vegetation above the level of the roof. The output is the 2D building model.

Figures 7 and 8 present the final result of automatic 2D building modelling for the Vaihingen and Hermanni sites respectively. Three colors are used in Figures $7 \mathrm{~b}, 7 \mathrm{~d}$ and $8 \mathrm{~b}$. The blue represents the building outer boundaries, the red represents the inner roof plane boundaries and the green represents the missing plane boundaries.

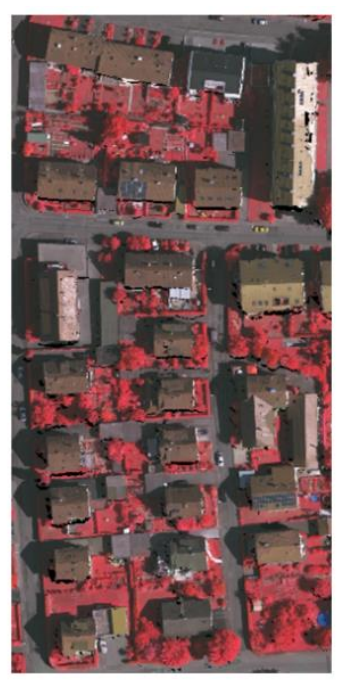

(a)

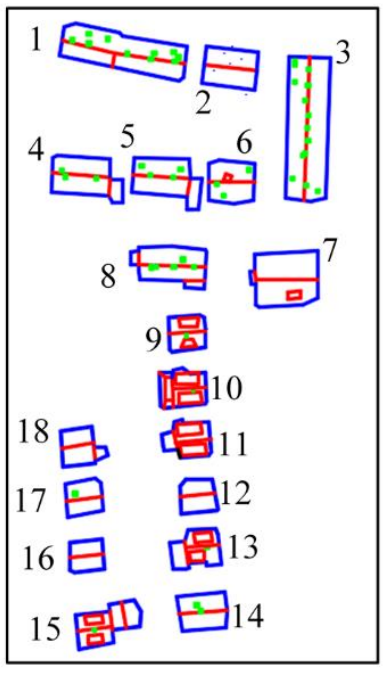

(b)

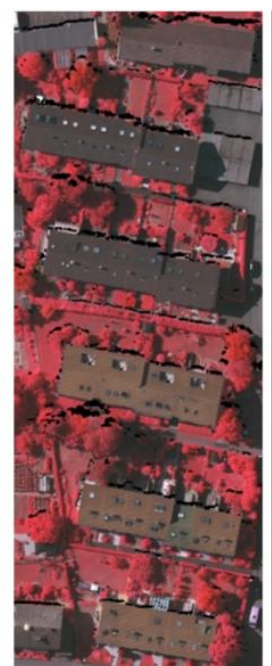

(c)

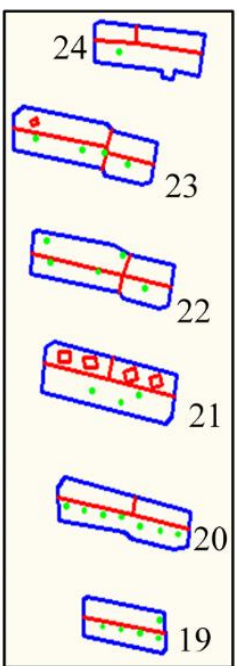

(d)

Figure 7. Final result of automatic 2D building modelling of Vaihingen site; (b) and (d) 2D building models; (a) and (c) Aerial images; Colors in (b) and (d): blue: building outer boundaries; red: inner roof plane boundaries; green: missing planes. 


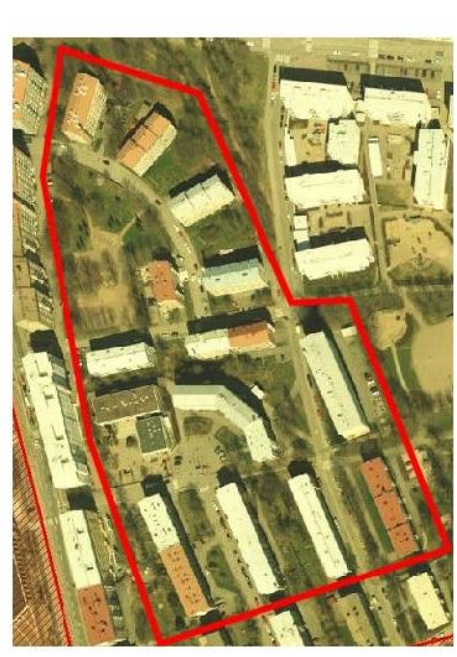

(a)

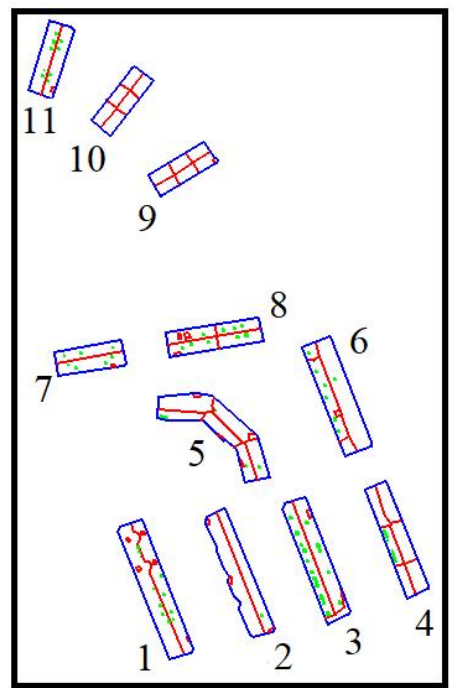

(b)

Figure 8. Final result of automatic 2D building modelling of Hermanni site; (a) Aerial images; (b) 2D building models; Colors in (b): blue: building outer boundaries; red: inner roof plane boundaries; green: missing planes.

It should be noted that roof details are sometimes represented in red when their boundaries are considered as inner roof boundaries. In this case, the roof detail is detected by the RANSAC algorithm through the segmentation of the building roof point cloud. In contrast, some roof details are represented by the color green because their areas are small, and consequently, they are not detected by the RANSAC algorithm through the segmentation of building roof point cloud. Fortunately, they are detected later by the missing planes modelling algorithm. Finally, qualitative analysis and quantification of the accuracy of the results are carried out as follows.

\subsection{Accuracy analysis}

In order to estimate the accuracy of building models calculated from Lidar data, Tarsha Kurdi, Awrangjeb, and Munir (2019), Dorninger and Pfeifer (2008), Ostrowski et al. (2018), Elberink and Vossleman (2011), Akca et al. (2010), Sampath and Shan (2010) and Park et al. (2019) 
suggest using the Lidar point cloud as reference data. This choice has been adopted because it allows the study of deformation generated only by the modelling algorithm independently of the quality of the input building point cloud. One of the crucial elements of these approaches of modelling accuracy estimation is the calculation of distances (point per point) between the roof planes and the point cloud. There are two main forms to present the accuracy estimation result: vector form (Ostrowski et al., 2018) and raster form (Tarsha Kurdi \& Awrangjeb, 2020). In this context, Tarsha Kurdi and Awrangjeb (2020) suggest the calculation of the SAQ (Segmentation Accuracy and Quality) factor based on the error map matrix (see Figure 9). Indeed, this matrix expresses the building model accuracy in comparison to the building point cloud. The pixel values in this matrix represent the distances (deviations) between each point and its mean corresponding plane. For example, if the pixel value is equal or close to zero, the model in this point is precise and vice versa. Furthermore, the missing roof planes appear clearly in building error map matrix (see Figure 9a). Therefore, the error map is also used for detecting and modelling the missing roof planes (see Section 5.2).

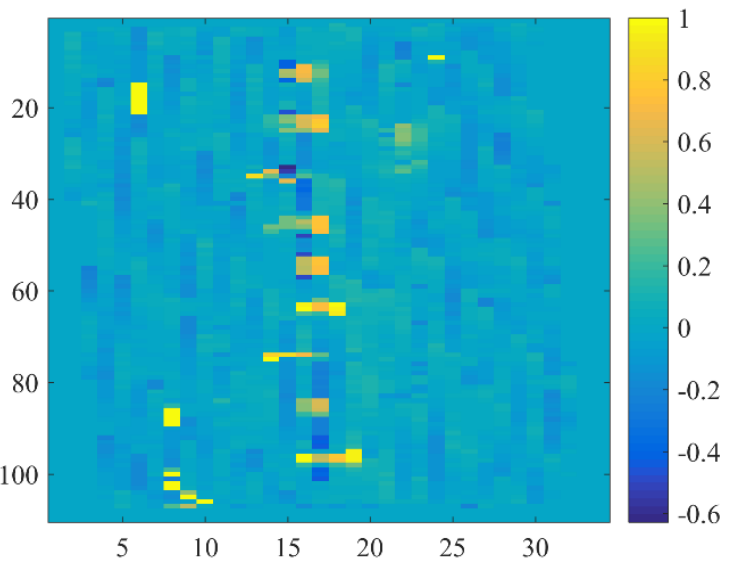

(a)

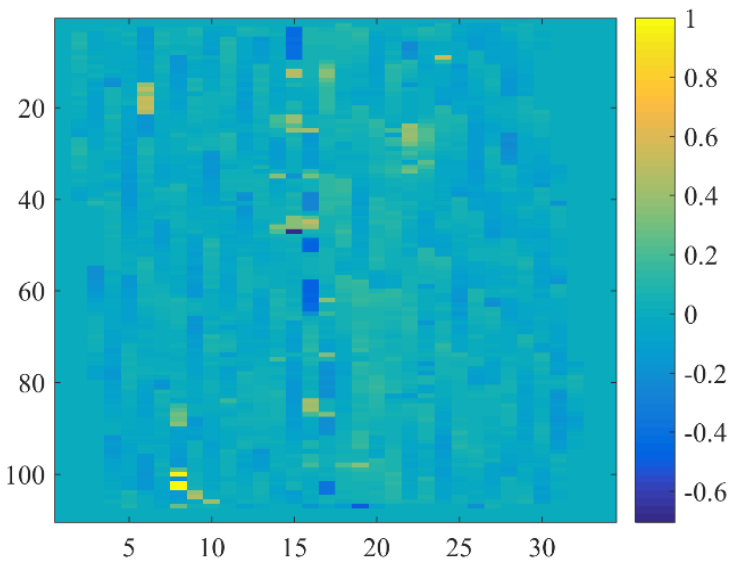

(b)

Figure 9. Visualization of error map matrix of Building 3 in Figure 7; (a) and (b) respectively before and after modelling of missing roof planes. 
In this paper, the approach suggested by Tarsha Kurdi and Awrangjeb (2020) is adopted because it not only links the missing planes modelling algorithm with the accuracy estimation, but it also estimates the accuracy due only to the modelling algorithm, without considering the quality of the input point cloud. In this approach, the SAQ factor value has to be calculated before and after the detection of missing planes. At this stage, it is important to note that the SAQ factor expresses the percentage of error map matrix pixels having values outside the interval [-0.25 m, +0.25 m] (Tarsha Kurdi \& Awrangjeb, 2020).

In order to estimate the accuracy of $2 \mathrm{D}$ building models presented in Figures $7 \mathrm{~b}, 7 \mathrm{~d}$, and $8 \mathrm{~b}$, the SAQ factors are calculated before and after the modelling of the missing roof planes (see Tables 5 and 6).

Table 5. Values of SAQ factor of Vaihingen building site before and after missing plane modelling

\begin{tabular}{|c|c|c|c|c|c|c|c|c|c|c|c|c|}
\hline $\begin{array}{c}\text { Building } \\
\text { number }\end{array}$ & 1 & 2 & 3 & 4 & 5 & 6 & 7 & 8 & 9 & 10 & 11 & 12 \\
\hline SAQ1(\%) & 2.39 & 1.57 & 4.01 & 2.40 & 2.91 & 1.81 & 1.18 & 3.16 & 0.89 & 2.09 & 1.67 & 1.79 \\
\hline SAQ2(\%) & 1.54 & 0.36 & 2.42 & 1.03 & 1.41 & 0.75 & 0.52 & 1.45 & -- & 1.57 & 0.67 & 1.53 \\
\hline $\begin{array}{c}\text { Building } \\
\text { number }\end{array}$ & 13 & 14 & 15 & 16 & 17 & 18 & 19 & 20 & 21 & 22 & 23 & 24 \\
\hline SAQ1(\%) & 0.66 & 1.77 & 0.96 & 3.50 & 2.58 & 2.69 & 0.82 & 1.30 & 1.16 & 1.70 & 0.58 & 0.15 \\
\hline SAQ2(\%) & -- & 1.14 & -- & 2.10 & 0.79 & 1.48 & -- & 0.12 & 0.06 & 0.10 & -- & -- \\
\hline
\end{tabular}

SAQ factor: Segmentation Accuracy and Quality.

Table 6. The values of SAQ factor before and after missing plane modelling of Hermanni site

\begin{tabular}{|c|c|c|c|c|c|c|c|c|c|c|c|c|}
\hline $\begin{array}{c}\text { Building } \\
\text { number }\end{array}$ & 1 & 2 & 3 & 4 & 5 & 6 & 7 & 8 & 9 & 10 & 11 & 12 \\
\hline SAQ1(\%) & 8.46 & 2.79 & 4.76 & 5.55 & 6.24 & 8.23 & 2.15 & 2.77 & 2.30 & 2.85 & 3.50 & 2.15 \\
\hline SAQ2(\%) & 0.94 & 0.92 & 2.03 & 1.64 & 1.55 & 3.11 & 0.35 & 0.35 & 1.37 & 0.70 & 0.87 & 0.35 \\
\hline
\end{tabular}

SAQ factor: Segmentation Accuracy and Quality.

In Tables 5 and 6, it can be noted that the buildings containing several missing roof planes have a relatively high value SAQ factor before modelling the missing planes (see Buildings 1 and 3 
in Table 5 and in Figure 7b). After modelling the missing planes, the SAQ factor value becomes smaller which means the accuracy of building models improves. Moreover, Buildings 9, 13, 15, 19, 23, and 24 in Table 5 have very accurate results before detecting the missing planes, because their SAQ factor values are smaller than $1 \%$. Therefore, the SAQ factors after detecting the missing planes are not calculated again. All constructed building models in Figures 7 and 8 have an excellent accuracy estimation regarding the value of the SAQ factor after the missing plane modelling (SAQ is smaller than 5\%; see Tarsha Kurdi and Awrangjeb, 2020). These results refer to the high quality of building roof segmentation and consequently the final building models.

The modelling accuracy is also tested through calculating the standard deviation of each 2D building model. For this goal, the pixel values in the error map matrix (see Figure 9) are used again because they present the deviations between the point cloud and the roof fitted planes. Figure 10 illustrates the standard deviations of $2 \mathrm{D}$ building models presented in Figures $7 \mathrm{~b}, 7 \mathrm{~d}$ and $8 \mathrm{~b}$ where 24 building models of the Vaihingen site are constructed and 12 building models of the Hermanni site are constructed. Figure 10 shows that the standard deviations are smaller than $0.2 \mathrm{~m}$, which can be considered as excellent results regarding the employed point cloud accuracy. Furthermore, it shows that the models of the Vaihingen site are more accurate than those of Hermanni site, despite the flight height of the Hermanni data (200 m) being lower than that of Vaihingen data $(500 \mathrm{~m})$. The reason for this accuracy difference comes from the quality of the Lidar sensor, which is related to the date of scanning (see Table 1). 


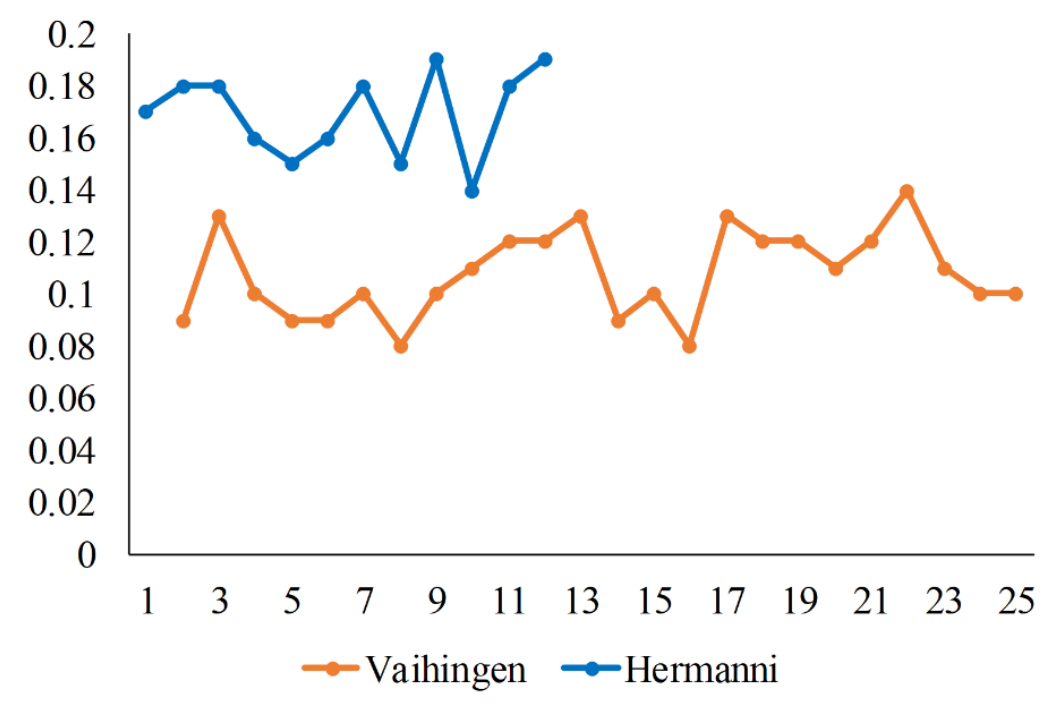

Figure 10. Standard deviations of building models.

Concerning the buildings presented in Figure $7 \mathrm{a}$, as their reference models are available (the ISPRS benchmark on urban object, Rottensteiner et al. 2014), the constructed models are compared with their reference models. For this purpose, three accuracy indices are calculated: The Completeness (Comp), the Correctness (Corr) and the Quality (Q). These indices count the number of roof planes that have an overlap of at least $50 \%$ with reference roof planes. For calculating these indices, the confusion matrix is employed (Shan, Yan, \& Jiang, 2018). In this context, three elements from the confusion matrix are employed: TP, FN and FP (see Section 4.5 and Equations 7, 8 and 9 by Rutzinger, Rottensteiner and Pfeifer (2009)). The average values of all considered buildings are shown in Table 7 . These indices are estimated two times. The first estimate is for all roof planes whereas the second is for only roof planes with areas greater than $10 \mathrm{~m}^{2}$.

$$
\begin{aligned}
& \operatorname{Comp}=\frac{\|T P\|}{\|T P\|+\|F N\|} \\
& \text { Corr }=\frac{\|T P\|}{\|T P\|+\|F P\|} \\
& Q=\frac{\|T P\|}{\|T P\|+\|F P\|+\|F N\|}
\end{aligned}
$$

The difference between the results in the two last cases is because the definitions of roof detail 
are different between the reference and the calculated models, i.e., in the constructed models, the roof detail (detectable or missing) has a significant height, but in the reference models which are constructed by the manual stereo plotting method, the operator has the freedom to decide if the roof detail is considerable or not. However, the results shown in Table 7 represent the efficiency of the suggested algorithm.

Table 7. Evaluation of the building reconstruction results: average of buildings in Figure 7a.

\begin{tabular}{|c|c|c|}
\hline Comp (\%) & Corr $(\%)$ & Q $(\%)$ \\
\hline 77.3 & 85.7 & 67.2 \\
\hline Comp $\left(10 \mathrm{~m}^{2}\right)(\%)$ & Corr $\left(10 \mathrm{~m}^{2}\right)(\%)$ & $\mathrm{Q}\left(10 \mathrm{~m}^{2}\right)(\%)$ \\
\hline 94.0 & 94.0 & 90.4 \\
\hline
\end{tabular}

The first estimate is for all roof planes, the second is for only roof planes with areas greater than $10 \mathrm{~m}^{2}$

Finally, sometimes it is observed that one building model contains some visual deformations despite its accuracy indexes being of high quality. In fact, the deformation of the building model may be due to the modelling algorithm limitation or/ and the building point cloud quality. Indeed, the point density, the point distribution heterogeneity, the position accuracy, architectural building complexity and the noise presence may generate deformation in the constructed model. Thus, the building model suffers from the accumulation of two types of deformations. The first one is due to the infidelity of point cloud toward the original building. The employed modelling algorithm generates the second type of deformations. When the error map matrix is used for accuracy estimation, only the deformations generated by the limitation of modelling algorithm are controlled. To summarize, there are three ordered models of each building: the original building, the point cloud and the calculated model. On the one hand, the calculated model has to be faithful to the building point cloud. On the other hand, it is necessary to estimate how much the point cloud is faithful to the original building. Consequently, more investigations are required in order to answer this question. 


\section{Conclusion and perspective}

This paper presented two linked topics in the context of automatic Lidar data processing. The first one is the automatic filtering of building point cloud for automatically detecting the roof point cloud which is the input data for the second topic. The second topic is the automatic construction of building footprint from Lidar data exclusively. The eliminating of the undesirable points from building point cloud helps to improve the quality of the constructed building model especially when the quantity of undesirable points is very high. In this context, the suggested approach assumes that the input building point cloud is free from the vegetation that occludes the roof segments. More investigations are envisaged in the future work for considering this case. For this purpose, the analysis of the normal vectors may be helpful. Another limitation of this algorithm is that it was tested only on the Lidar point clouds. Therefore, it has to be tested and extended in future research for other point cloud types such as the photogrammetric point clouds from the oblique areal imaging or from the Unmanned Aerial Vehicle (UAV) videos.

In the modelling operation, the generalization level of the constructed model is related directly to four factors: the point density, the points position accuracy, the cloud homogeneity level, and the architectural building complexity. At this stage, the error map matrix can play an important role for judging if the complete 2D building model is faithful to the input building point cloud or not. Moreover, it is employed for modelling the missing roof planes.

However, several adjustments were achieved to improve the quality of the $2 \mathrm{D}$ building model. In future research, this model can be refined by introducing certain geometric constraints, such as parallelism or orthogonality. Furthermore, profiting from the histogram of $Z$ coordinate of building roof point cloud may improve the quality of the building model. These improvements may make the building model closer to reality. 


\section{Acknowledgement:}

The Vaihingen data set was provided by the German Society for Photogrammetry, Remote Sensing and Geoinformation (DGPF) (Cramer, 2010): http://www.ifp.uni-stuttgart.de/dgpf/DKEP-Allg.html. This work represents an extension of our paper (Tarsha Kurdi, Awrangjeb, \& Munir, 2019).

\section{References}

Akca, D., Freeman, M., Sargent, I., \& Gruen, A. (2010, December 22). Quality assessment of 3D building data. The Photogrammetric Record, 25(132), pp. 339-355.

Ameri, B. (2000). Automatic recognition and 3D reconstruction of buildings from digital imagery, $\mathrm{PhD}$ thesis, German Geodetic Commission (DGK)-C 526, Institute for Photogrammetry, Stuttgart University.

Awrangjeb, M. (2016, January 21). Using point cloud data to identify, trace and regularize the outlines of buildings. International Journal of Remote Sensing, pp. 551-579, doi:10.1080/01431161.2015.1131868.

Awrangjeb, M., Gilani, S.A.N., \& Siddiqui, F.U. (2018, September 21). An effective datadriven method for 3D building roof reconstruction and robust change detection. Remote Sens 2018, 10, 1512, https://doi.org/10.3390/rs10101512.

Cramer, M. (2010, May 01). The DGPF test on digital aerial camera evaluation - overview and test design. Photogrammetrie - Fernerkundung - Geoinformation 2(2010), pp. 73-82. 
Demir, N. (2018, July 23). Automated detection of 3D roof planes from Lidar data. Journal of the Indian Society of Remote Sensing. August 2018, 46(8), pp. 1265-1272. https://doi.org/10.1007/s12524-018-0802-2.

Dorninger, P., \& Pfeifer, N. (2008, November 17). A comprehensive automated 3D approach for building extraction, reconstruction, and regularization from airborne laser scanning point clouds. Sensors, 8(11), doi: 10.3390/s8117323, pp.7323-7343.

Elberink, S.O., \& Vosselman. G. (2011, March). Quality analysis on 3D building models reconstructed from airborne laser scanning data. ISPRS Journal of Photogrammetry and Remote Sensing, 66(2), pp.157-165. http://doi.org/10.1016/j.isprsjprs.2010.09.009.

Ghaffarian, S., Ghaffarian, S., Elmerabet, Y., Samir, Z., \& Ruicheck, Y. (2016, October 1721). Automatic building roof segmentation based on PFICA algorithm and morphological filtering from Lidar point clouds. In 37th Asian Conference on Remote Sensing (ACRS 2016), promoting spatial data infrastructure for sustainable economic development, Colombo, Sri Lanka.

Goebbels, S., \& Pohle-Fröhlicha, R. (2016, July 12-19). Roof reconstruction from airborne laser scanning data based on image processing methods. ISPRS Annals of the Photogrammetry, Remote Sensing and Spatial Information Sciences, Volume III-3, 2016, XXIII ISPRS Congress, Prague, Czech Republic, doi :10.5194/isprsannals-III-3-407-2016, pp.407-414.

Gross, H., \& Thoennessen, U. (2006, September 20-22). Extraction of lines from laser point cloud. Int. Arch. of the Photogrammetry, Remote Sensing and Spatial Information Sciences, 
Proceedings of the ISPRS Commission III Symposium on Photogrammetric and Computer Vision, Volume XXXVI, Bonn, Germany, pp. 86-91.

Jaya, S.N., Akshay, J., \& Beena, K. (2018, May 30). Contour Extraction in Buildings in Airborne Lidar Point Clouds Using Multiscale Local Geometric Descriptors and Visual Analytics. IEEE Journal of Selected Topics in Applied Earth Observations and Remote Sensing, doi: 10.1109/JSTARS.2018.2833801, PP. 1-16.

Jung, J., \& Sohn, G. (2018). Progressive modeling of 3D building rooftops from airborne Lidar and imagery. Topographic Laser Ranging and Scanning: Principles and Processing (2nd ed.). Shan, J., \& Toth, C.K. Eds: CRC Press: Boca Raton, FL, USA, pp. 523-562.

Li, M., Rottensteiner, F., \& Heipke, C. (2019, June 11). Modelling of buildings from aerial Lidar point clouds using TINs and label maps. ISPRS Journal of Photogrammetry and Remote Sensing, 154, https://doi.org/10.1016/j.isprsjprs.2019.06.003, pp. 127-138.

Martin-Jimenez, J., Del Pozo, S., Sanchez-Aparicio, M., \& Laguela, S. (2020, January). Multiscale roof characterization from Lidar data and aerial orthoimagery: Automatic computation of building photovoltaic capacity. Journal Automation in Construction, Volume 109, 102965, https://doi.org/10.1016/j.autcon.2019.102965.

Ostrowski, W., Pilarska, M., Charyton, J., \& Bakuła, K. (2018, June 4-7). Analysis of 3D building models accuracy based on the airborne Laser scanning point clouds. Int. Arch.of the Photogrammetry, Remote Sensing and Spatial Information Sciences, Volume XLII-2, 2018, 
ISPRS TC II Mid-term Symposium “Towards Photogrammetry 2020”, Riva del Garda, Italy. https://doi.org/10.5194/isprs-archives-XLII-2-797-2018.

Park, S.Y., Lee, D.G., Yoo, E.J., \& Lee, D.C. (2019, April 17). Segmentation of Lidar data using multilevel cube code. Journal of Sensors, Volume 2019, Article ID 4098413, 18 pages, https://doi.org/10.1155/2019/4098413.

Perera, S.N., Nalani, H.A., \& Maas, H.G. (2012, July 20). An automated method for 3D roof outline generation and regularization in airborne laser scanner data. Int. Arch. of the Photogrammetry, Remote Sensing, ISPRS Ann. Photogrammetry. Remote Sens. Spatial Inform. Sci. I-3, pp. 281-286.

Pirotti, F., Zanchetta, C., Previtali, M., \& Della Torre, S. (2019, May 8-10). Detection of building roofs and facades from aerial laser scanning data using deep learning. ISPRS- Int. Arch.of the Photogrammetry, Remote Sensing and Spatial Information Sciences 4211 (2019), https://doi.org/10.5194/isprs-archives-XLII-2-W11-975- 2019, pp. 975-980.

Rottensteiner, F., Trinder, J., Clode, S., \& Kubik, K. (2005, September 12-14). Automated delineation of roof planes from Lidar data. Int. Arch. of the Photogrammetry, Remote Sensing, ISPRS WG III/3, III/4, V/3 Workshop "Laser scanning 2005", Enschede, Netherlands, 2005. pp. 221-226.

Rottensteiner, F. (2003, November 30). Automatic generation of high-quality building models from Lidar data. IEEE CG\&A, 23(6), dio: 10.1109/MCG.2003.1242381, pp. 42-50. 
Rottensteiner, F., Sohn, G., Gerke, M., Wegner, J. D., Breitkopf, U., \& Jung, J. (2014, July). Results of the ISPRS benchmark on urban object detection and 3D building reconstruction. ISPRS Journal of Photogrammetry and Remote Sensing, 93(2014), http://dx.doi.org/10.1016/j.isprsjprs.2013.10.004, pp.256-271.

Rutzinger, M., Rottensteiner, F., \& Pfeifer, N. (2009, March). A comparison of evaluation techniques for building extraction from airborne laser scanning. IEEE Journal of Selected Topics in Applied Earth Observations \& Remote Sens. 2 (1), dio: 10.1109/JSTARS. 2009. 2012488, pp.11-20.

Sampath, A., \& Shan, J. (2006, May 1-5). Clustering based planar roof extraction from Lidar data. American Society for Photogrammetry and Remote Sensing, Annual Conference, Reno, Nevada.

Sampath, A., \& Shan, J. (2010, March). Segmentation and reconstruction of polyhedral building roofs from aerial Lidar point clouds. IEEE transactions on geoscience and remote sensing, vol. 48, no. 3, dio: 10.1109/TGRS.2009.2030180, pp. 1554 -1567.

Schenk, T., \& Csatho, B. (2002). Fusion of Lidar data and aerial imagery for a more complete surface description. Int. Arch.of the Photogrammetry, Remote Sensing and Spatial Information Sciences, Proceedings of the ISPRS Commission III Symposium on Photogrammetric and Computer Vision, Volume XXXIV, Graz, Austria, 2002, pp. 310-317. 
Serratosa, F., \& Sanfeliu, A. (2006, January 21). Signatures versus histograms: definitions, distances and algorithms. Pattern Recognition https://doi.org/10.1016/j.patcog.2005.12.005, pp. 921 - 934.

Shan, J., Yan, J., \& Jiang, W. (2018). Global solutions to building segmentation and reconstruction. Topographic Laser Ranging and Scanning: Principles and Processing (2nd ed.). Shan, J.; Toth, \& C.K., Eds: CRC Press, Boca Raton, FL, USA, 2018, pp. 459-484.

Sohn, G., \& Dowman, I. (2007, February 15). Data fusion of high-resolution satellite imagery and Lidar data for automatic building extraction. ISPRS Journal of Photogrammetry and Remote Sensing, 62(1), May, https://doi.org/10.1016/j.isprsjprs.2007.01.001, pp. 43-63.

Sohn, G., Huang, X., \& Tao, V. (2008, November 01). Using a binary space partitioning tree for reconstructing polyhedral building models from airborne Lidar data. Photogrammetric Engineering and Remote Sensing, $\quad$ Number https://doi.org/10.14358/PERS.74.11.1425, pp. 1425-1438(14).

Tarsha Kurdi, F., \& Awrangjeb, M. (2020, March 01). Automatic evaluation and improvement of roof segments for modelling missing details using Lidar data. International Journal of Remote $\quad$ Sensing, $\quad$ Volume $\quad 41, \quad 2020 \quad$ - Issue $\quad 12$, https://doi.org/10.1080/01431161.2020.1723180, pp. 4702-4725.

Tarsha Kurdi, F., Awrangjeb, M., \& Munir, N. (2019, September 26-27). Automatic 2D modelling of inner roof planes boundaries starting from Lidar data. 14th 3D GeoInfo 2019, Singapore, doi: 10.5194/isprs-annals-IV-4-W8-107-2019, pp.107-114. 
Tarsha Kurdi, F., Landes, T., \& Grussenmeyer, P. (2007, April 11-13). Joint combination of point cloud and DSM for 3D building reconstruction using airborne laser scanner data. 4th IEEE GRSS / WG III/2+5, VIII/1, VII/4 Joint Workshop on Remote Sensing \& Data Fusion over Urban Areas and 6th International Symposium on Remote Sensing of Urban Areas. Télécom Paris, doi : 10.1109/URS.2007.371843, IEEE, 7 p.

Tarsha Kurdi, F., Landes, T., Grussenmeyer, P., \& Koehl, M. (2007, September19-21). Modeldriven and data-driven approaches using Lidar data: analysis and comparison. Int. Arch.of the Photogrammetry, Remote Sensing, PIA07, Photogrammetry Image Analysis. Munich, Germany, pp.87-92.

Tarsha Kurdi, F., Landes, T., \& Grussenmeyer, P. (2008). Extended RANSAC algorithm for automatic detection of building roof planes from Lidar data. The Photogrammetric Journal of Finland, vol. 21, n¹, 2008, pp. 97-109.

Vosselman, G., \& Dijkman, S. (2001, October 22-24). 3D building model reconstruction from point clouds and ground plans. Int. Arch. of the Photogrammetry, Remote Sensing, XXXIV3/W4, pp. 37-43.

Xiao, Y., Wang, C., Li, J., Zhang, W., Xi, X., Wang, C., \& Dong, P. (2015, May 14). Building segmentation and modelling from airborne Lidar data, International Journal of Digital Earth, 8:9, doi: 10.1080/17538947.2014.914252, pp. 694-709. 
Xiong, B., Jancosek, M., Oude Elberink S., \& Vosselman G. (2015, January 30). Flexible building primitives for 3D building modeling. ISPRS Journal of Photogrammetry and Remote Sensing 101 (2015), http://dx.doi.org/10.1016/j.isprsjprs.2015.01.002, pp. 275-290.

Zhang, K., Yan, J., \& Chen, S.C. (2018). A framework for automated construction of building models from airborne Lidar measurements. Topographic Laser Ranging and Scanning: Principles and Processing (2nd ed.). Shan, J., \& Toth, C.K. Eds: CRC Press, Boca Raton, FL, USA, 2018, pp. 563-586.

Zhang, W., Wang, H., Chen, Y., Yan, K., \& Chen, M. (2014, August 28). 3D building roof modeling by optimizing primitive's parameters using constraints from Lidar data and aerial imagery. Remote Sens. 2014, 6(9), doi:10.3390/rs6098107. pp. 8107-8133. 